JOURNAL OF

APPLIED CRYSTALLOGRAPHY

ISSN 1600-5767

Received 8 September 2015

Accepted 2 November 2015

Edited by S. Sasaki, Tokyo Institute of

Technology, Yokohama, Japan

Keywords: equivalent description of crystal structures; normalizers; normal subgroups; conjugated subgroups; crystallographic education.

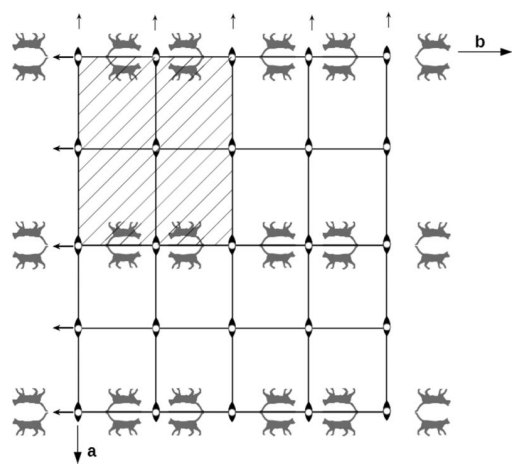

C 2015 International Union of Crystallography

\section{The same once again please: a pedagogical introduction to normalizers}

\author{
Massimo Nespolo* \\ Université de Lorraine, CRM2, UMR 7036, Vandoeuvre-les-Nancy, F-54506, France, and CNRS, CRM2, UMR 7036, \\ Vandoeuvre-les-Nancy, F-54506, France. *Correspondence e-mail: massimo.nespolo@crm2.uhp-nancy.fr
}

Normalizers of space groups are extensively used in the solution of diverse tasks in structural crystallography. Yet, they are often considered an 'esoteric' topic, seldom introduced in the education curriculum of a crystallographer. This paper presents a pedagogical introduction with special emphasis on their role in the equivalent description of crystal structures.

\section{Introduction}

Normalizers of space groups represent an extremely useful tool for the crystallographer, intervening in many diverse tasks of the investigation of the structure and properties of condensed matter. Some of these tasks, like the solution of the ambiguities in direct methods, are often transparent to the final user, being instead of paramount importance, for example, for crystallographic software developers. But, other problems that have to be dealt with directly by the structural crystallographer, like the equivalent description of crystal structures, are solved by the use of normalizers (Hahn, 2005). ${ }^{\mathbf{1}}$ Despite some excellent literature on the subject (e.g. Koch \& Fischer, 2006), the experience of running crystallographic schools in the framework of the activities of the IUCr Commission on Mathematical and Theoretical Crystallography (Nespolo \& McColm, 2014) has shown that this is one of the topics that the participants find most difficult to understand. Today we have access to online tools like the Bilbao Crystallographic Server (Aroyo et al., 2006), where not only the normalizers of any space group can be retrieved, but the comparison of crystal structure descriptions equivalent under the action of the normalizer can actually be performed in a few clicks (Tasci et al., 2012). However, without getting a precise idea of what these mathematical objects are, how they work and what they are useful for, the whole set of tools may remain a sort of black box, and the results obtained may not be straightforward to interpret, possibly giving rise to misunderstandings and false statements in the application of these results to one's own structural work. Without the pretension of giving a complete formal introduction to normalizers, which is already available in the literature, in the following we try to present an intuitive picture which will hopefully be of some help in overcoming the barrier too often felt by newcomers when they first meet this topic. We will start with point groups, where the general principles are easier to illustrate and understand, before moving to space groups, with the final aim

\footnotetext{
${ }^{1}$ For the sake of brevity, the different chapters of Volume $A$ of International Tables for Crystallography will be quoted below as ITA $X$, where ' $X$ ' is the number of the chapter.
} 
of showing how to obtain the equivalent descriptions of the same crystal structure.

\section{Point groups}

We start this brief analysis with point groups, whose properties are easily understood in terms of the stereographic projection of face forms. About the stereographic projection, see Whittaker (1984) or, for a detailed treatment, Terpstra \& Codd (1961). For crystal forms, see ITA10 and a recent discussion by Nespolo (2015a).

\subsection{Conjugated subgroups versus normal subgroups}

Fig. 1 part (a) shows the stereographic projection of a general $\{h k l\}$ form in point group ..2. Part $(b)$ shows the result of the application of a reflection in the $m_{[010]}$ plane to the form in part $(a)$. Parts $(c)$ and $(d)$ show the result of the application of $4_{[001]}^{1}$ rotation to the forms in parts $(a)$ and $(b)$, respectively. The four stereographic projections are different but their point group is the same: the object (the form) is changed (i.e. is

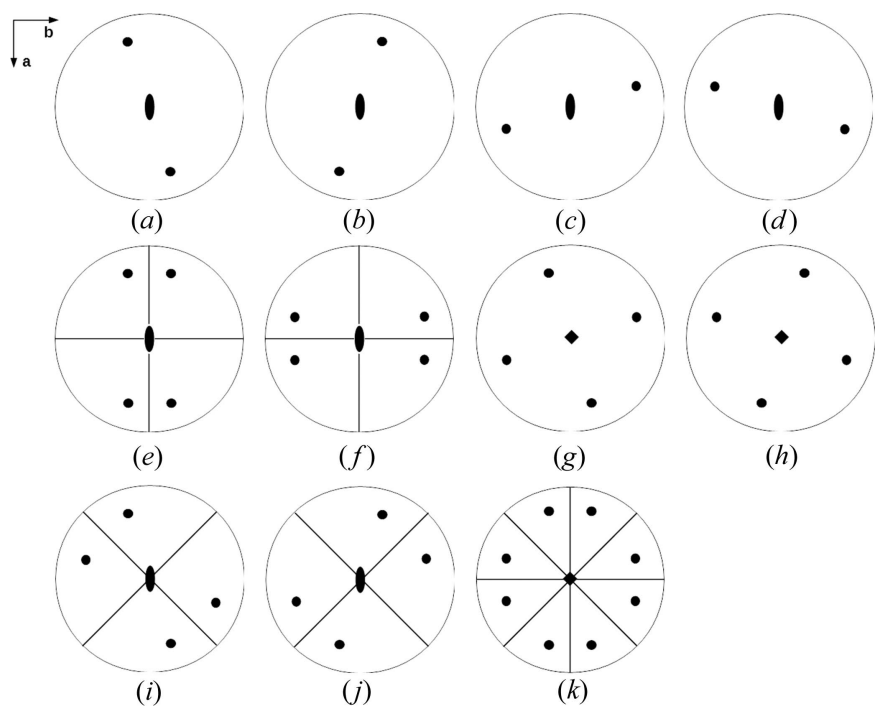

Figure 1

(a) Stereographic projection of a general $\{h k l\}$ form in point group ..2. (b) Reflection across the (010) plane applied to $(a)$. (c) Fourfold vertical rotation applied to $(a)$. $(d)$ Fourfold vertical rotation applied to $(b)$. The four projections all have point group ..2. (e) Overlap of $(a)$ and $(b)$ leading to a form with point group $m m 2$. $(f)$ Overlap of $(c)$ and $(d)$ leading to a form with point group $m m 2$. The operations in $m m 2$ not contained in. .2 map directly $(a)$ to $(b)$ and $(c)$ to $(d)$ and map by conjugation the respective point groups, which in this case coincide. $(g)$ Overlap of $(a)$ and $(c)$ leading to a form with point group 4. (h) Overlap of $(b)$ and $(d)$ leading to a form with point group 4 . The operations in 4 not contained in ..2 map directly $(a)$ to $(c)$ and $(b)$ to $(d)$ and map by conjugation the respective point groups, which coincide. (i) Overlap of $(a)$ and $(d)$ leading to a form with point group 2.mm. $(j)$ Overlap of $(b)$ and (c) leading to a form with point group 2. $\mathrm{mm}$. The operations in $2 . \mathrm{mm}$ not contained in. .2 map directly $(a)$ to $(d)$ and $(b)$ to $(c)$ and map by conjugation the respective point groups, which coincide. $(k)$ Overlap of $(a),(b),(c)$ and $(d)$ leading to a form with point group $4 m m$. The operations in $4 \mathrm{~mm}$ not contained in ..2 map directly $(a)$ to $(b),(c)$ and $(d)$ and map by conjugation the respective point groups, which coincide. not invariant under the operation), because that operation does not belong to the point group of the object, whereas the point group of the object remains the same (i.e. is invariant under the same operation).

Parts $(e)$ and $(f)$ show the compound forms obtained by the overlap, respectively, of $(a)$ and $(b)$, and of $(c)$ and $(d)$. In both cases the resulting point group is $m m 2$, which is the supergroup of 2 containing also the operations mapping the forms $(a)$ to $(b)$ and $(c)$ to $(d)$. The two compound forms are different but they have the same point group.

Parts $(g)$ and $(h)$ show the compound forms obtained by the overlap, respectively, of $(a)$ and $(c)$, and of $(b)$ and $(d)$. In both cases the resulting point group is 4 , which is the supergroup of 2 containing also the operations mapping the forms $(a)$ to $(c)$ and $(b)$ to $(d)$. As in the previous case, the forms are different but they have the same point group.

Parts $(i)$ and $(j)$ show the compound forms obtained by the overlap, respectively, of $(a)$ and $(d)$, and of $(b)$ and $(c)$. In both cases the resulting point group is $m m 2$ as for parts $(e)$ and $(f)$, but here the mirrors are rotated $45^{\circ}$. To differentiate the two groups it is customary to use for the latter the symbol $2 . \mathrm{mm}$, which makes reference to a tetragonal setting: the dot in the second position means that there is no symmetry element along the $a$ and $b$ axes. $2 . \mathrm{mm}$ is the supergroup of 2 containing also the operations mapping the forms $(a)$ to $(d)$ and $(b)$ to $(c)$. Once again, the forms are different but they have the same point group.

Finally, part $(k)$ is obtained as the overlap of $(g)$ and $(h)$, or of $(i)$ and $(j)$. The resulting point group is $4 \mathrm{~mm}$, which is the supergroup of 2 containing also all the operations mapping the form $(a)$ to $(b),(c)$ and $(d)$.

Fig. 2 repeats the same scheme as Fig. 1 but now on a general $\{h k l\}$ form in point group $m$.. For parts $(a)$ and $(b)$ the same considerations presented in the case of Fig. 1 apply here too. Things change when we apply the vertical fourfold rotation. The point group of the forms in parts $(c)$ and $(d)$ is now different from the point group of the forms in parts $(a)$ and (b): it contains the same type of operations as the former, but the reflection is performed about a geometric element (the plane acting as a mirror, in this case) differently oriented in space. Parts $(e)$ and $(f)$ show the compound forms, whose point group is $m m 2$, i.e. the supergroup of $m$.. and of.$m$. containing also the operations mapping the forms $(a)$ to $(b)$ and $(c)$ to $(d)$.

The forms in parts $(g)-(j)$, obtained in the same way as the corresponding forms in Fig. 1, deserve special consideration. The point group compatible with each of these projections is ..m (expressed with respect to the tetragonal setting). Neither the nontrivial symmetry operations of the original forms $(m$.. and.$m$. in the orthorhombic setting) nor the isometries mapping them (the fourfold rotations) appear in the compound projections because they do not apply to the whole set of poles but only to pairs. In other words, these operations are not global but partial (Sadanaga et al., 1980). Only the operation obtained as a combination of $m$.. (or .m.), and $4^{1}$ (or $\left.4^{3}\right)$, is a global operation. We will come back to this aspect shortly. 
The complete compound projection is the same as in Fig. $1(k)$ and has point group $4 m m$, in which all the above operations are global. $4 \mathrm{~mm}$ is in fact the supergroup of both $m$.. and .m. which contains also the operations mapping $(a)$ to $(b),(c)$ and $(d)$.

The point group $m$.. of $(a)$ or $(b)$ is invariant under the point group $m m 2$ of $(e)$ or $(f)$, and the same is true for the point group .m. of $(c)$ or $(d)$, but they are not invariant under the point group $4 \mathrm{~mm}$ of $(\mathrm{k})$; actually, the operations of $4 \mathrm{~mm}$ that do not belong to $m m 2$ relate $m$.. and.$m$.

Let us see how we can rationalize the above examples and let us denote by $\mathrm{o}_{1}, \mathrm{o}_{2}, \ldots, \mathrm{o}_{n}$ the $n$ isometries that map a form $\mathrm{F}$ to a form $\mathrm{F}^{\prime}$. We want to find how to formally describe the action of these operations on the form $\mathrm{F}$ as well as on its group $\mathcal{G}$. The $i$ th operation $\mathrm{o}_{i}$ will be applied to $\mathrm{F}$ from the left, so that the action is written as

$$
\mathrm{o}_{i} \mathrm{~F}=\mathrm{F}^{\prime}, \quad \mathrm{o}_{i}^{-1} \mathrm{~F}^{\prime}=\mathrm{F},
$$

where $\mathrm{o}_{i}^{-1}$ is the inverse of $\mathrm{o}_{i}$. In our example above, $i=1$ corresponds to $2_{[001]}$ (Fig. 1) or to $m_{[010]}$ (Fig. 2), and $i=2$ to $4_{\text {[001] }}$ (both figures). If $g$ is a given operation of the point group $\mathcal{G}$ of $\mathrm{F}$, which implies that $\mathrm{g} F=\mathrm{F}$ because $\mathrm{F}$ is invariant under $g$, and $g^{\prime}$ is a given operation of the point group $\mathcal{G}^{\prime}$ of $\mathrm{F}^{\prime}$, which implies that $g^{\prime} \mathrm{F}^{\prime}=\mathrm{F}^{\prime}$ because $\mathrm{F}^{\prime}$ is invariant under $g^{\prime}$, the following relations are immediately obtained from equation (1):

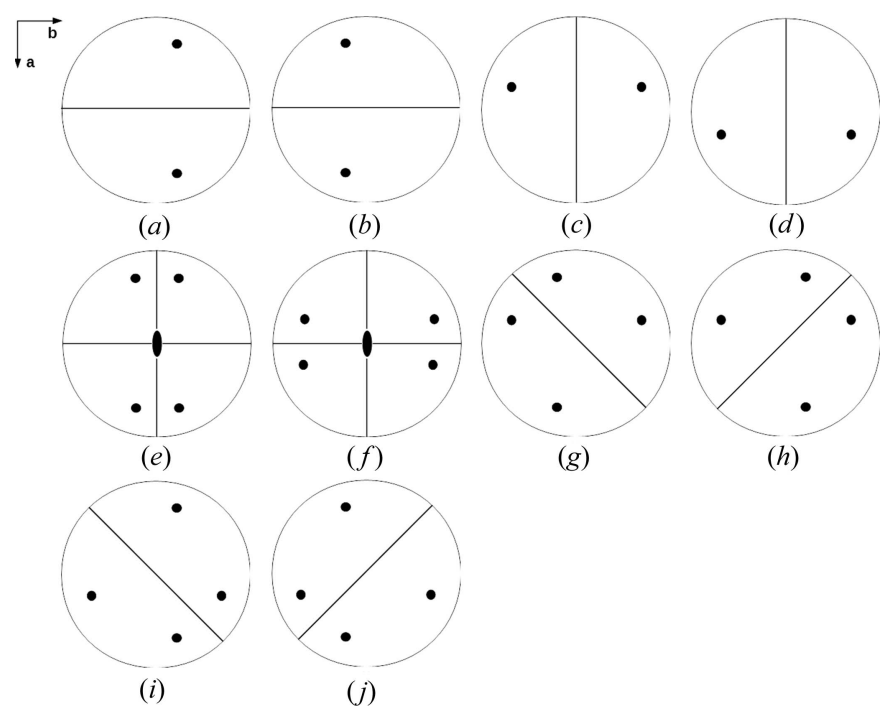

Figure 2

(a) Stereographic projection of a general $\{h k l\}$ form in point group $m$. (b) Reflection across the (010) plane applied to $(a)$. The two projections have point group $m . .(c)$ Fourfold vertical rotation applied to $(a)$. $(d)$ Fourfold vertical rotation applied to $(b)$. The two projections have point group.$m$. $(e)$ Overlap of $(a)$ and $(b)$ leading to a form with point group $m m 2 .(f)$ Overlap of $(c)$ and $(d)$ leading to a form with point group $m m 2$. The operations in $m m 2$ not contained in .m. map directly $(a)$ to $(b)$ and the operations in $m m 2$ not contained in $m$.. map directly $(c)$ to $(d)$; they map by conjugation the respective point groups, which in this case do not coincide. ( $g$ ) Overlap of $(a)$ and $(c)$. (h) Overlap of $(b)$ and $(d)$. (i) Overlap of $(a)$ and $(d)$. ( $j)$ Overlap of $(b)$ and $(c)$. These four forms have as nontrivial symmetry operation a reflection across a diagonal plane: the reflections in the original forms as well as the fourfold rotation do not appear in the point group of the compound form because they act only as partial operations.

$$
\begin{aligned}
& \mathrm{o}_{i} \mathrm{~F}=\mathrm{o}_{i}(g \mathrm{~F})=\left(\mathrm{o}_{i} g\right) \mathrm{F}=\mathrm{F}^{\prime}=g^{\prime} \mathrm{F}^{\prime}, \\
& \mathrm{o}_{i} \mathrm{~F}=\left(\mathrm{o}_{i} g\right) \mathrm{o}_{i}^{-1} \mathrm{~F}^{\prime}=\mathrm{F}^{\prime}=g^{\prime} \mathrm{F}^{\prime}, \\
& \mathrm{o}_{i} g \mathrm{o}_{i}^{-1} \mathrm{~F}^{\prime}=g^{\prime} \mathrm{F}^{\prime}, \quad \mathrm{o}_{i}^{-1} g^{\prime} \mathrm{o}_{i} \mathrm{~F}=g \mathrm{~F} .
\end{aligned}
$$

The relation $(2 c)$ tells us that a symmetry operation $g^{\prime}$ of $F^{\prime}$ can be expressed in terms of a symmetry operation $g$ of $F$ (or vice versa) by acting on it with an isometry mapping $\mathrm{F}$ to $\mathrm{F}^{\prime}$. This transformation is known as conjugation and is a similarity transformation, i.e. a shape-preserving transformation. We see that while the operation $\mathrm{o}_{i}$ acts directly on an object (the form $\mathrm{F})$, it acts by conjugation on the symmetry group of that object. In the example above, the projection in Fig. $2(a)$ is mapped to that in Fig. $2(c)$ by the $4^{1}$ (or $4^{3}$ ) rotation and the nontrivial symmetry operation of $(a)$ is conjugated to the nontrivial symmetry operation of $(c)$ :

$$
4_{[001]}^{1} m_{[100]} 4_{[001]}^{3}=m_{[010]} .
$$

In general, $g$ and $g^{\prime}$ may or may not coincide; for example, if instead of $m_{[100]}$ in the relation above we take the identity, then $g^{\prime}$ is also the identity.

When the index $i$ is made to run over all the $n$ isometries mapping $\mathrm{F}$ to $\mathrm{F}^{\prime}$, we obtain all the operations $g^{\prime}$ that are conjugated to a given $g$ by these isometries:

$$
\cup_{i=1}^{n} \mathrm{o}_{i} g \mathrm{o}_{i}^{-1}=\left\{g_{1}^{\prime}, g_{2}^{\prime}, \ldots, g_{n}^{\prime}\right\},
$$

where $\cup$ means set-theoretical union (i.e. the collection of the operations identified by the running index $i$ ). If the result is $n$ times $g$, then one says that $g$ is self-conjugated under the $n$ isometries mapping $\mathrm{F}$ to $\mathrm{F}^{\prime}$.

All the operations in equation (4) are of the same type as $g$. In fact, a point symmetry operation is represented by an $m \times$ $m$ invertible matrix, where $m$ is the dimension of the space, and the successive application of symmetry operations is represented by matrix multiplication. If $\mathbf{O}_{i}$, $\mathbf{G}$ and $\mathbf{G}^{\prime}$ represent the $i$ operations $\mathrm{o}_{i}, g$ and $g^{\prime}$, respectively, equation $(2 c)$ is rewritten in terms of the matrices representing these operations as

$$
\mathbf{O}_{i} \mathbf{G O}_{i}^{-1}=\mathbf{G}^{\prime}
$$

Now, a matrix possesses two invariants under a similarity transformation: determinant and trace, which are scalars and identify uniquely the type of operation the matrix represents. That the determinant is indeed invariant is evident from the following relation:

$$
\operatorname{det}\left(\mathbf{O}_{i} \mathbf{G O}_{i}^{-1}\right)=\operatorname{det}\left(\mathbf{O}_{i}\right) \operatorname{det}(\mathbf{G}) \operatorname{det}\left(\mathbf{O}_{i}^{-1}\right)=\operatorname{det}\left(\mathbf{G}^{\prime}\right) .
$$

Because scalars commute and $\operatorname{det}\left(\mathbf{M}^{-1}\right)=1 / \operatorname{det}(\mathbf{M})$, where $\mathbf{M}$ is any invertible matrix,

$$
\begin{aligned}
\operatorname{det}\left(\mathbf{O}_{i}\right) \operatorname{det}(\mathbf{G}) \operatorname{det}\left(\mathbf{O}_{i}^{-1}\right) & =\operatorname{det}(\mathbf{G}) \operatorname{det}\left(\mathbf{O}_{i}\right) \operatorname{det}\left(\mathbf{O}_{i}^{-1}\right) \\
& =\operatorname{det}(\mathbf{G}) \operatorname{det}\left(\mathbf{O}_{i}\right)\left[1 / \operatorname{det}\left(\mathbf{O}_{i}\right)\right] \\
& =\operatorname{det}(\mathbf{G})=\operatorname{det}\left(\mathbf{G}^{\prime}\right) .
\end{aligned}
$$

A similar result is obtained for the trace, by exploiting the property that the trace is invariant under commutation:

$$
\operatorname{Tr}\left(\mathbf{O}_{i} \mathbf{G} \mathbf{O}_{i}^{-1}\right)=\operatorname{Tr}\left(\mathbf{G O} \mathbf{O}_{i}^{-1}\right)=\operatorname{Tr}(\mathbf{G})=\operatorname{Tr}\left(\mathbf{G}^{\prime}\right) .
$$


Therefore, equation $(2 c)$ relates operations of the same type and all the operations in equation (4) are of the same type. This is often translated in everyday language by saying that conjugation 'does the same thing somewhere else', as in the example of Figs. 2(a) and $2(d)$.

Next, let us apply equation (4) not to a single symmetry operation $g$ of $\mathrm{F}$ but to the whole set of its symmetry operations, i.e. to the group $\mathcal{G}$ of F. Equation (4) is immediately generalized:

$$
\cup_{i=1}^{n} \mathrm{o}_{i} \mathcal{G} \mathrm{o}_{i}^{-1}=\mathcal{G}^{\prime} .
$$

The group $\mathcal{G}^{\prime}$ is isomorphic to $\mathcal{G}$; in fact

(a) 1 (the identity operation) belongs to both $\mathcal{G}$ and $\mathcal{G}^{\prime}$ because, for any value of $i, \mathrm{o}_{i} 1 \mathrm{o}_{i}^{-1}=1 \mathrm{o}_{i} \mathrm{o}_{i}^{-1}=1$;

$(b)$ the conjugate of the inverse of each operation $g$ belongs to $\mathcal{G}^{\prime}$.

Let us indicate as $g_{j}$ and $g_{k}$ two operations of which one is the inverse of the other in $\mathcal{G}$. This means that $g_{j} g_{k}=1$. We can exploit the result above as follows:

$$
\begin{aligned}
\mathrm{o}_{i} 1 \mathrm{o}_{i}^{-1} & =\mathrm{o}_{i} g_{j} g_{k} \mathrm{o}_{i}^{-1}=\mathrm{o}_{i} g_{j} 1 g_{k} \mathrm{o}_{i}^{-1}=\mathrm{o}_{i} g_{j}\left(\mathrm{o}_{i}^{-1} \mathrm{o}_{i}\right) g_{k} \mathrm{o}_{i}^{-1} \\
& =\left(\mathrm{o}_{i} g_{j} \mathrm{o}_{i}^{-1}\right)\left(\mathrm{o}_{i} g_{k} \mathrm{o}_{i}^{-1}\right)=g_{j}^{\prime} g_{k}^{\prime},
\end{aligned}
$$

where we have made use of the fact that the identity operation 1 can always be written as the product of two inverse operations. Because the left-hand member is the identity, the righthand member is also the identity and thus $g_{j}^{\prime} g_{k}^{\prime}$ are the inverse of each other, which proves the statement.

The groups $\mathcal{G}$ and $\mathcal{G}^{\prime}$ are thus conjugated under the isometries mapping $\mathrm{F}$ to $\mathrm{F}^{\prime}$ : they contain the same type of operations, but in general about geometric elements differently oriented in space. In the case of the projections in Figs. 2(a) and 2(c) equation (9) becomes

$$
4_{[001]}^{1}\left\{1, m_{[100]}\right\} 4_{[001]}^{3} \cup 4_{[001]}^{3}\left\{1, m_{[100]}\right\} 4_{[001]}^{1}=\left\{1, m_{[010]}\right\}
$$

by taking only once each operation obtained by conjugation. Not surprisingly, we get the same result as we knew from the stereographic projections. To obtain a general relation between $\mathcal{G}$ and $\mathcal{G}^{\prime}$ we need to find the supergroup $\mathcal{K}$ common to both groups which contains the isometries mapping $\mathrm{F}$ to $\mathrm{F}^{\prime}$ : $\mathcal{K}$ is the symmetry group of the compound form. This is obtained by taking the set-theoretical union of the group with the sets of operations obtained by combining the group operations and the independent isometries $\mathrm{o}_{i}$, which can however be applied to the group from the left or from the right:

$$
\mathcal{K}=\mathcal{G} \cup_{i} \mathrm{o}_{i} \mathcal{G}=\mathcal{G} \cup_{i} \mathcal{G} \mathrm{o}_{i}=\mathcal{G}^{\prime} \cup_{i} \mathrm{o}_{i} \mathcal{G}^{\prime}=\mathcal{G}^{\prime} \cup_{i} \mathcal{G}^{\prime} \mathrm{o}_{i},
$$

where $i$ is now a running index on the independent isometries mapping $\mathrm{F}$ and $\mathrm{F}^{\prime} . \mathrm{o}_{i} \mathcal{G}$ is called a left coset, and $\mathcal{G}_{\mathrm{o}_{i}}$ a right coset, of $\mathcal{K}$. The decomposition of $\mathcal{K}$ into cosets is a partition: the elements of $\mathcal{K}$ are separated in subsets (the subgroup and the cosets) without a common element. The number of elements in a coset is the length of that coset: it cannot be called the order because a coset is not a group (it does not contain the identity). The decomposition into left or right cosets does not give, in general, the same result: elements that belong to the same left coset may appear in different right cosets and vice versa. Let us see how concretely it works with the examples in Figs. 1 and 2.

In Fig. $1, \mathcal{G}=\mathcal{G}^{\prime}=\left\{1,2_{[001]}\right\}$. The group $\mathcal{K}$ of the compound forms $(e)$ or $(f)$ is obtained by taking the set-theoretical union of $\mathcal{G}$ and $m_{[010]} \mathcal{G}$ or $\mathcal{G} m_{[010]}$ :

$$
\begin{aligned}
\mathcal{G} \cup m_{[010]} \mathcal{G} & =\left\{1,2_{[001]}\right\} \cup m_{[010]}\left\{1,2_{[001]}\right\} \\
& =\left\{1,2_{[001]}\right\} \cup\left\{m_{[010]}, m_{[100]}\right\}=m m 2, \\
\mathcal{G} \cup \mathcal{G} m_{[010]} & =\left\{1,2_{[001]}\right\} \cup\left\{1,2_{[001]}\right\} m_{[010]} \\
& =\left\{1,2_{[001]}\right\} \cup\left\{m_{[010]}, m_{[100]}\right\}=m m 2 .
\end{aligned}
$$

The group $\mathcal{K}$ of the compound forms $(g)$ or $(h)$ is obtained by taking the set-theoretical union of $\mathcal{G}$ and $4_{[001]} \mathcal{G}$ or $\mathcal{G} 4_{[001]}$ :

$$
\begin{aligned}
\mathcal{G} \cup 4_{[001]} \mathcal{G} & =\left\{1,2_{[001]}\right\} \cup 4_{[001]}\left\{1,2_{[001]}\right\} \\
& =\left\{1,2_{[001]}\right\} \cup\left\{4_{[001]}, 4_{[001]}^{3}\right\}=4, \\
\mathcal{G} \cup \mathcal{G} 4_{[001]} & =\left\{1,2_{[001]}\right\} \cup\left\{1,2_{[001]}\right\} 4_{[001]} \\
& =\left\{1,2_{[001]}\right\} \cup\left\{4_{[001]}, 4_{[001]}^{3}\right\}=4 .
\end{aligned}
$$

The group $\mathcal{K}$ of the compound forms $(i)$ or $(j)$ is obtained by taking the set-theoretical union of $\mathcal{G}$ and $4_{[001]} m_{[010]} \mathcal{G}$ $\left(=m_{[1 \overline{1} 0]} \mathcal{G}\right)$ or $\mathcal{G} m_{[010]}{ }_{[001]}\left(=\mathcal{G} m_{[110]}\right)$ :

$$
\begin{aligned}
\mathcal{G} \cup 4_{[001]} m_{[010]} \mathcal{G} & =\left\{1,2_{[001]}\right\} \cup m_{[1 \overline{10}]}\left\{1,2_{[001]}\right\} \\
& =\left\{1,2_{[001]}\right\} \cup\left\{m_{[1 \overline{1} 0]}, m_{[110]}\right\}=2 . m m, \\
\mathcal{G} \cup \mathcal{G} m_{[010]} 4_{[001]} & =\left\{1,2_{[001]}\right\} \cup\left\{1,2_{[001]}\right\} m_{[110]} \\
& =\left\{1,2_{[001]}\right\} \cup\left\{m_{[110]}, m_{[1 \overline{1} 0]}\right\}=2 . m m .
\end{aligned}
$$

Finally, the group $\mathcal{K}$ of the compound form $(k)$ is obtained by taking the set-theoretical union of the results above:

$$
\begin{aligned}
\mathcal{G} & \cup m_{[010]} \mathcal{G} \cup 4_{[001]} \mathcal{G} \cup 4_{[001]} m_{[010]} \mathcal{G} \\
& =\left\{1,2_{[001]}\right\} \cup m_{[010]}\left\{1,2_{[001]}\right\} \cup 4_{[001]}\left\{1,2_{[001]}\right\} \cup m_{[1 \overline{10}]}\left\{1,2_{[001]}\right\} \\
& =\left\{1,2_{[001]}\right\} \cup\left\{m_{[010]}, m_{[100]}\right\} \cup\left\{4_{[001]}, 4_{[001]}^{3}\right\} \cup\left\{m_{[1 \overline{10}]}, m_{[110]}\right\} \\
& =4 m m, \\
\mathcal{G} & \cup \mathcal{G} m_{[010]} \cup \mathcal{G} 4_{[001]} \cup \mathcal{G} m_{[010]} 4_{[001]} \\
& =\left\{1,2_{[001]}\right\} \cup\left\{1,2_{[001]}\right\} m_{[010]} \cup\left\{1,2_{[001]}\right\} 4_{[001]} \cup\left\{1,2_{[001]}\right\} m_{[110]} \\
& =\left\{1,2_{[001]}\right\} \cup\left\{m_{[010]}, m_{[100]}\right\} \cup\left\{4_{[001]}, 4_{[001]}^{3}\right\} \cup\left\{m_{[110]}, m_{[1 \overline{10}]}\right\} \\
& =4 m m .
\end{aligned}
$$

We can repeat the step-by-step procedure above for the forms in Fig. 2, but in the case of the forms in parts $(g)-(j)$, where partial operations are involved, we do not obtain a group. For the forms in part $(g)$ or $(h)$ we obtain

$$
\begin{aligned}
\mathcal{G} \cup 4_{[001]} \mathcal{G} & =\left\{1, m_{[100]}\right\} \cup 4_{[001]}\left\{1, m_{[100]}\right\} \\
& =\left\{1, m_{[100]}\right\} \cup\left\{4_{[001]}, m_{[110]}\right\}, \\
\mathcal{G} \cup \mathcal{G}_{[001]} & =\left\{1, m_{[100]}\right\} \cup\left\{1, m_{[100]}\right\} 4_{[001]} \\
& =\left\{1, m_{[100]}\right\} \cup\left\{4_{[001]}, m_{[1 \overline{1} 0]}\right\},
\end{aligned}
$$


and for the forms in parts $(i)$ or $(j)$,

$$
\begin{aligned}
\mathcal{G}^{\prime} \cup 4_{[001]} \mathcal{G}^{\prime} & =\left\{1, m_{[010]}\right\} \cup 4_{[001]}\left\{1, m_{[010]}\right\} \\
& =\left\{1, m_{[010]}\right\} \cup\left\{4_{[001]}, m_{[110]}\right\}, \\
\mathcal{G}^{\prime} \cup \mathcal{G}^{\prime} 4_{[001]} & =\left\{1, m_{[010]}\right\} \cup\left\{1, m_{[010]}\right\} 4_{[001]} \\
& =\left\{1, m_{[010]}\right\} \cup\left\{4_{[001]}, m_{[110]}\right\},
\end{aligned}
$$

none of which is a group (the set is not closed under successive applications of the operations in the set). The fact that $m_{[110]}$ or $m_{[1 \overline{1} 0]}$ is actually a global operation is not evident and requires a more specific analysis [a detailed example is given by Nespolo \& Aroyo (2015)].

The point group of the complete form, which is the same as in Fig. $1(k)$, is obtained by collecting the results for the intermediate forms:

$$
\begin{aligned}
\mathcal{G} \cup & m_{[010]} \mathcal{G} \cup 4_{[001]} \mathcal{G} \cup 4_{[001]} m_{[010]} \mathcal{G}=\left\{1, m_{[100]}\right\} \\
& \cup m_{[010]}\left\{1, m_{[100]}\right\} \cup 4_{[001]}\left\{1, m_{[100]}\right\} \cup m_{[1 \overline{10}]}\left\{1, m_{[100]}\right\} \\
= & \left\{1, m_{[100]}\right\} \cup\left\{m_{[010]}, 2_{[001]}\right\} \cup\left\{4_{[001]}, m_{[110]}\right\} \cup\left\{m_{[1 \overline{10}]}, 4_{[001]}^{3}\right\} \\
= & 4 m m,
\end{aligned}
$$

$$
\begin{aligned}
\mathcal{G} \cup & \mathcal{G} m_{[010]} \cup \mathcal{G} 4_{[001]} \cup \mathcal{G} m_{[010]} 4_{[001]}=\left\{1, m_{[100]}\right\} \\
& \cup\left\{1, m_{[100]}\right\} m_{[010]} \cup\left\{1, m_{[100]} 4_{[001]} \cup\left\{1, m_{[100]}\right\} m_{[110]}\right. \\
= & \left\{1, m_{[100]}\right\} \cup\left\{m_{[010]}, 2_{[001]}\right\} \cup\left\{4_{[001]}, m_{[110]}\right\} \cup\left\{m_{[110]}, 4_{[001]}^{3}\right\} \\
= & 4 m m,
\end{aligned}
$$

but this time the third and fourth coset are different. The same procedure applied to $\mathcal{G}^{\prime}$ leads to the same result:

$$
\begin{aligned}
& \mathcal{G}^{\prime} \cup m_{[100]} \mathcal{G}^{\prime} \cup 4_{[001]} \mathcal{G}^{\prime} \cup 4_{[001]} m_{[100]} \mathcal{G}^{\prime}=\left\{1, m_{[010]}\right\} \\
& \cup m_{[100]}\left\{1, m_{[010]}\right\} \cup 4_{[001]}\left\{1, m_{[010]}\right\} \cup m_{[110]}\left\{1, m_{[010]}\right\} \\
&=\left\{1, m_{[100]}\right\} \cup\left\{m_{[100]}, 2_{[001]}\right\} \cup\left\{4_{[001]}, m_{[110]}\right\} \cup\left\{m_{[110]}, 4_{[001]}^{3}\right\} \\
&= 4 m m,
\end{aligned}
$$

$$
\begin{aligned}
& \mathcal{G}^{\prime} \cup \mathcal{G}^{\prime} m_{[100]} \cup \mathcal{G}^{\prime} 4_{[001]} \cup \mathcal{G}^{\prime} m_{[100]} 4_{[001]}=\left\{1, m_{[010]}\right\} \\
& \cup\left\{1, m_{[010]}\right\} m_{[100]} \cup\left\{1, m_{[010]}\right\} 4_{[001]} \cup\left\{1, m_{[010]}\right\} m_{[1 \overline{1} 0]} \\
&=\left\{1, m_{[010]}\right\} \cup\left\{m_{[100]}, 2_{[001]}\right\} \cup\left\{4_{[001]}, m_{[110]}\right\} \cup\left\{m_{[1 \overline{1} 0]}, 4_{[001]}^{3}\right\} \\
&= 4 m m .
\end{aligned}
$$

$\mathcal{K}$ is thus the supergroup common to $\mathcal{G}$ and $\mathcal{G}^{\prime}$ obtained by adding the isometries that conjugate them; $\mathcal{G}$ and $\mathcal{G}^{\prime}$ are therefore conjugated subgroups of $\mathcal{K}$ and they contain the same type of symmetry operations but about geometric elements in general differently oriented in space. The mapping of $\mathcal{G}$ to $\mathcal{G}^{\prime}$, or vice versa, is obtained by the action of symmetry operations that belong to $\mathcal{K}$ but not to $\mathcal{G}$ or $\mathcal{G}^{\prime}$. If $\mathcal{G}$ coincides with $\mathcal{G}^{\prime}$, as in the case of Fig. 1, then it is self-conjugated, i.e. invariant, under any of the operations of $\mathcal{K}$. $\mathcal{G}$ is then called an invariant or normal subgroup of $\mathcal{K}$.

We obtain the following conclusions:

(1) The operations of $\mathcal{K}$ applied to $\mathrm{F}$ produce $n$ variants, $\mathrm{F}_{1}$ to $\mathrm{F}_{n}$, not necessarily all different, where $n$ is the index of $\mathcal{G}$ in $\mathcal{K}$ and $\mathcal{G}$ is the point group of $\mathrm{F}$.
(2) The operations of $\mathcal{K}$ transform the group $\mathcal{G}$ of $\mathrm{F}$ into the group $\mathcal{G}_{j}$ of $\mathrm{F}_{j}$ by conjugation, and the groups obtained are conjugated subgroups of $\mathcal{K}$. If they are all identical, then $\mathcal{G}$ is an invariant or normal subgroup of $\mathcal{K}$ and is conventionally indicated as $\mathcal{G} \triangleleft \mathcal{K}$.

(3) From the viewpoint of the matrices representing the operations acting on the form [equation (5)], conjugation is simply the transformation to a different basis. When $\mathcal{G}$ is a normal subgroup of $\mathcal{K}$, then conjugation of $\mathcal{G}$ by an operation of $\mathcal{K}$ is a change of coordinate system which respects the geometric elements of $\mathcal{G}$.

\subsection{The concept of normalizer}

Let us consider a group $\mathcal{K}$, a subgroup $\mathcal{G}$ of $\mathcal{K}$, and an intermediate subgroup $\mathcal{M}$ so that $\mathcal{G}$ is normal in $\mathcal{M}, \mathcal{G} \triangleleft \mathcal{M}$. $\mathcal{M}$ may actually coincide with $\mathcal{K}$ (in which case $\mathcal{G}$ is normal in $\mathcal{K}: \mathcal{G} \triangleleft \mathcal{K}$ ) or with $\mathcal{G}$ (in which case $\mathcal{G}$ is not normal in $\mathcal{K}$ or the intermediate subgroups, if any). $\mathcal{M}$ is called the normalizer of $\mathcal{G}$ with respect to $\mathcal{K}$, which is indicated as $\mathcal{M}=\mathcal{N}_{\mathcal{K}}(\mathcal{G})$. Because $\mathcal{G}$ is normal in $\mathcal{N}_{\mathcal{K}}(\mathcal{G})$, all the operations of $\mathcal{N}_{\mathcal{K}}(\mathcal{G})$ leave $\mathcal{G}$ invariant, i.e. the symmetry groups of the objects obtained by applying the operation of $\mathcal{N}_{\mathcal{K}}(\mathcal{G})$ to an object whose symmetry group is $\mathcal{G}$ are all identical to $\mathcal{G}$.

Let us then consider the largest possible $\mathcal{K}$, i.e. the orthogonal group $\mathrm{O}(n, \mathbf{R})$. This is the subgroup of the Euclidean group (which contains all the isometries in the given space $\mathbf{R}^{n}$ ) which leaves a point fixed, chosen as the origin of the space. It represents the symmetry of the sphere. The normalizer of $\mathcal{G}$ with respect to $\mathrm{O}(n, \mathbf{R}), \mathrm{N}_{\mathrm{O}}(\mathcal{G})$, contains all the origin-fixing isometries that leave $\mathcal{G}$ invariant. If one applies the operations of $\mathrm{O}(n, \mathbf{R})$ to an object, the result is the set of all possible orientations of that object; the symmetry group of each of these object is conjugated in $\mathrm{O}(n, \mathbf{R})$ with respect to $\mathcal{G}$, i.e. it contains the same operations as $\mathcal{G}$ but performed 'somewhere else' (about geometric elements differently oriented in space). If instead one applies the operations of $\mathrm{N}_{\mathrm{O}}(\mathcal{G})$ to the same object, the result is the set of all possible orientations of that object which have the same symmetry group as the original object.

The normalizers of point groups are given in $\S 4$ of ITA15. For example, for the point group $\mathcal{G}=m m 2$ which is one of the point groups of the compound forms discussed above, the normalizer is $\mathrm{N}_{\mathrm{O}}(\mathcal{G})=4 / \mathrm{mmm}$. The orders of the two groups are 4 and 16 , respectively, so that $\mathcal{G}$ has index four in $\mathrm{N}_{\mathrm{O}}(\mathcal{G})$. The decomposition of $\mathrm{N}_{\mathrm{O}}(\mathcal{G})$ with respect to $\mathcal{G}$ gives $\mathcal{G}$ itself and three cosets, each of length four, and thus four possible orientations for $\mathrm{F}$, all having $\mathcal{G}$ as point group.

For the point group $\mathcal{G}=m$, the normalizer is $\mathrm{N}_{\mathrm{O}}(\mathcal{G})=$ $\infty / m 2 / m$, one of the Curie groups (ITA10) corresponding to the symmetry of a stationary cylinder. The rotation axis $\infty$, which allows arbitrary rotations, is perpendicular to the mirror plane in $\mathcal{G}$; the mirror plane normal to the $\infty$ rotation axis coincides with the mirror plane in $\mathcal{G}$; the infinite set of twofold rotation axes in $\mathrm{N}_{\mathrm{O}}(\mathcal{G})$ is perpendicular to the $\infty$ rotation axis and all these axes are contained in the mirror plane of $\mathcal{G}$; the infinite set of mirror planes in $\mathrm{N}_{\mathrm{O}}(\mathcal{G})$ is perpendicular to the 
mirror plane of $\mathcal{G}$. The orders of the two groups are 4 and $\infty$, respectively, so that $\mathcal{G}$ has infinite index in $\mathrm{N}_{\mathrm{O}}(\mathcal{G})$. The decomposition of $\mathrm{N}_{\mathrm{O}}(\mathcal{G})$ with respect to $\mathcal{G}$ gives $\mathcal{G}$ itself and an infinite number of cosets, each of length four, and thus infinite possible orientations for $\mathrm{F}$, each having the same symmetry group as the generating form. In the stereographic projection the corresponding poles build a cylindrical orbit about the rotation axis of $\mathrm{N}_{\mathrm{O}}(\mathcal{G})$. $^{2}$

\section{Space groups}

The general ideas developed in dealing with point groups are easily extended to space groups. However, in moving from point groups to space groups the global picture becomes more complex because of the presence of translations and the consequences of their presence. Similarly to what we have done in \$2.1 regarding forms and their point groups, we present the effect of isometries on the symmetry of a crystallographic pattern and the resulting symmetry group obtained by overlapping these patterns. All the isometries we are going to use preserve the space group of the pattern, so that it is invariant under the symmetry group of the overlapped patterns; the latter is thus the normalizer of the space group of the pattern.

\subsection{A feline introduction to normalizers of space groups}

The conclusions obtained from equations (5), (7) and (8) apply to the linear part of the symmetry operations of a space group. The translation part is transformed according to the new orientation of the symmetry element. For example, an $a_{[010]}$ glide reflection becomes a $b_{[100]}$ glide reflection under the action of a $4_{[001]}$ rotation.

Fig. 3 shows a crystal pattern composed of cats in a space group of type $P c a 2_{1}$. Cats of different colours (black or white) are related by operations of the second kind (negative determinant). By applying a mirror reflection $m x y 0$ one gets the pattern in Fig. 4, where the height and the colour of the cats have been inverted. The space group of this second pattern is still $P c a 2_{1}$. By comparing the two figures, it is easy to understand that the two patterns are actually two different descriptions of the same pattern. In fact, if we move the origin of the second pattern by $2 z, z$ being the height of the black cat close to the origin in the first pattern, the only difference left between the two patterns is the colour. The difference of colour indicates a possible difference of handedness (if the cats are chiral), but there is a degree of freedom in attributing either the black or the white colour to a cat chosen as representative (for example the one closest to the origin). What is important is the relation between the colours of pairs of cats, which indicates whether two cats are related by an operation of the first kind (cats of the same colour) or of the second kind (opposite colour).

\footnotetext{
${ }^{2}$ The term orbit describes any set of objects that are mapped onto each other by the action of a group. Given a group $\mathcal{G}$ and a point $\mathrm{p}$, the set of all points $\mathcal{G}$ p obtained by applying all the operations of $\mathcal{G}$ to $\mathrm{p}$ constitutes an orbit of which $\mathcal{G}$ is called the generating group.
}

Figs. 3 and 4 are only two among the many possible equivalent descriptions of the same pattern. To find all of them, one proceeds to identify the normalizer of the space group. This time, however, also the translations have to be considered, so that not just the origin-preserving orthogonal group $\mathrm{O}(n, \mathbf{R})$, as was the case for the point groups, but the whole Euclidean group $\mathrm{E}(n)$, which includes the translations, has to be considered. As a first step, let us consider the overlap of the two patterns obtained so far (Fig. 5). A dichromatic cat

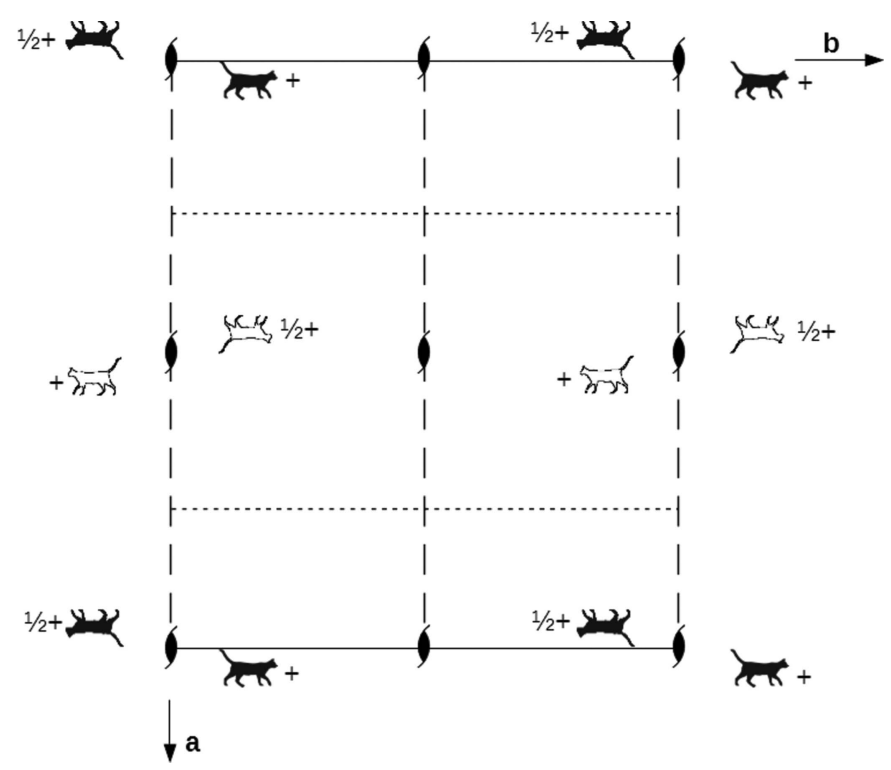

Figure 3

A crystal pattern composed of cats in a space group of type $P c a 2_{1}$. Cats of different colours (black or white) are related by operations of the second kind. Origin at the top-left corner of the diagram (same choice of origin in the following figures).

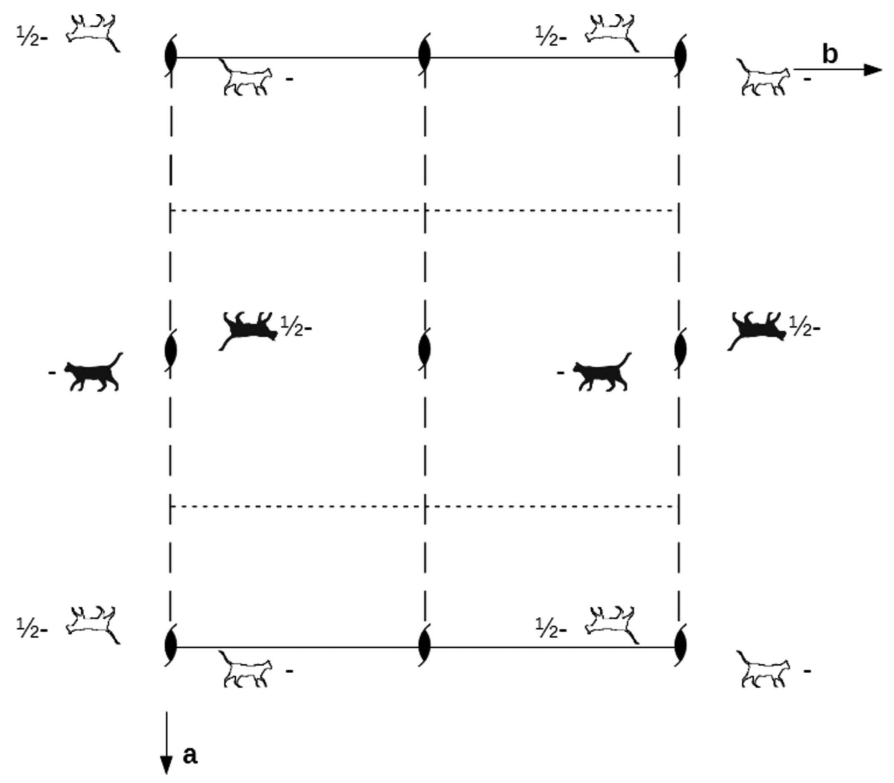

Figure 4

Result of the application of a mirror reflection $m x y 0$ to the pattern in Fig. 3. The space group of this pattern is still $P c a 2_{1}$ and the two patterns are actually two different descriptions of the same pattern, differing in the absolute height of the whole pattern and in the inversion of the colours. 
indicates the overlap in projection of two cats related by a second-kind operation; the cat closer to the observer has been cut in half (no harm meant, the writer being a proved ailurophile), leaving only the head side; in this way, the tail side of the cat farther from the observer is visible. The space group of this compound pattern is obtained exactly as in the example of point groups [equation (12)] and is of type $P 2_{1} / c 2 / a 2_{1} / m$, which is a nonstandard setting of $P 2 / b 2_{1} / c 2_{1} / m$ (No. 57) with axial transformation $-\mathbf{b}, \mathbf{a}, \mathbf{c}+\frac{1}{4}$.

Next, we can apply a translation of $\mathbf{a} / 2$, or of $\mathbf{b} / 2$, to the result obtained so far. The result for the $\mathbf{b} / 2$ translation is shown in Fig. 6; that for $\mathbf{a} / 2$ is analogous, differing only in the direction of the translation. Quite obviously, this is once again the same pattern, in an alternative but equivalent description. Let us then overlap the pattern in Fig. 5 with that obtained by b/2 translation (Fig. 6): the result is shown in Fig. 7 and the space group of this pattern is an isomorphic supergroup of $P 2_{1} / c 2 / a 2_{1} / m$ whose translational symmetry is doubled along $\mathbf{b}$. Let us then overlap the result just obtained with the same pattern translated by a/2 (Fig. 8): the space group of this pattern is $P 2 / c 2 / m 2_{1} / m$ with basis vectors $\mathbf{a} / 2, \mathbf{b} / 2$, c with respect to the $P c a 2_{1}$ space group of the pattern in Fig. 3. $P 2 / c 2 / m 2_{1} / m$ is a nonstandard setting of $P 2_{1} / m 2 / m 2 / a$ (No. 51) with axial transformation $\mathbf{c}, \mathbf{b},-\mathbf{a}$.

The result obtained is not yet the Euclidean normalizer of $P c a 2_{1}$. The latter is in fact a pyroelectic group containing a polar direction (the $c$ axis) along which the origin is undetermined. This means that there are an infinite number of equivalent descriptions of the same pattern obtained by

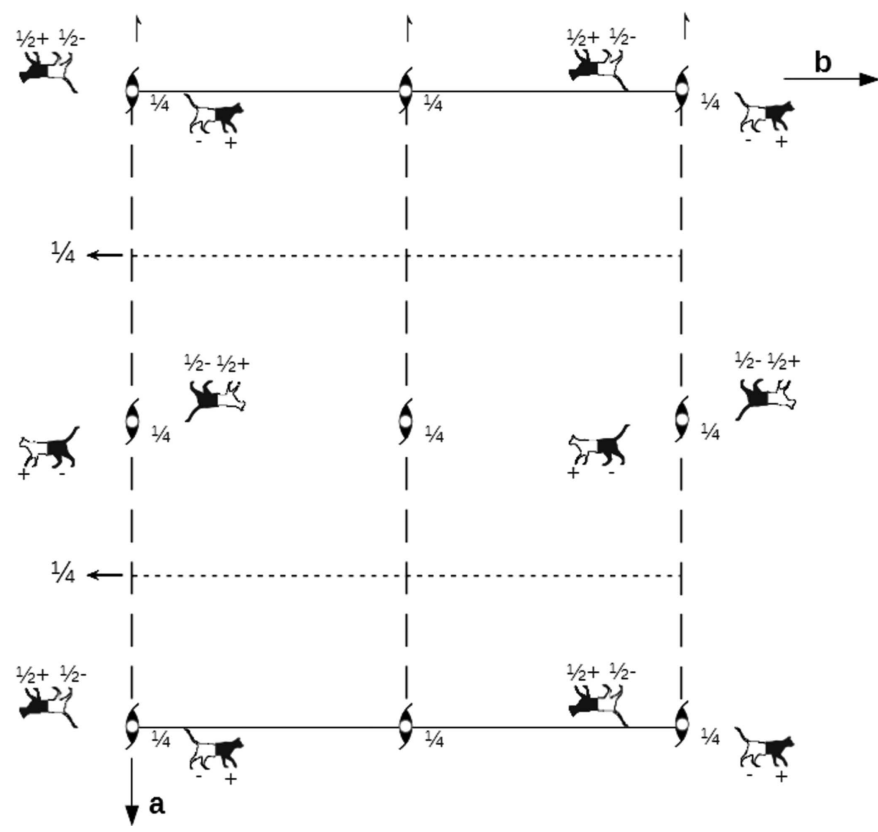

Figure 5

Overlap of the two patterns in Figs. 3 and 4. The dichromatic cat indicates the overlap in projection of two cats related by a second-kind operation; the cat closer to the observer has been cut in half, leaving only the head side; the tail side of the cat farther from the observer is visible. The space group of this compound pattern is obtained by extending $P c a 2_{1}$ by the $m_{0 y z}$ reflection and is of type $P 2_{1} / c 2 / a 2_{1} / m$, which is a nonstandard setting of $P 2 / b 2_{1} / c 2_{1} / m$ (No. 57) with axial transformation $-\mathbf{b}, \mathbf{a}, \mathbf{c}+\frac{1}{4}$. simply applying an infinitesimal translation along c. If we collect all these patterns in a single figure, we obtain, for each cat position in Fig. 8, a grey (black and white at the same time) feline continuity along c (Fig. 9). In this pattern, which presents an infinitesimal periodicity along $\mathbf{c}$, there is no screw or glide component for the symmetry operations: the $2_{1[001]}$

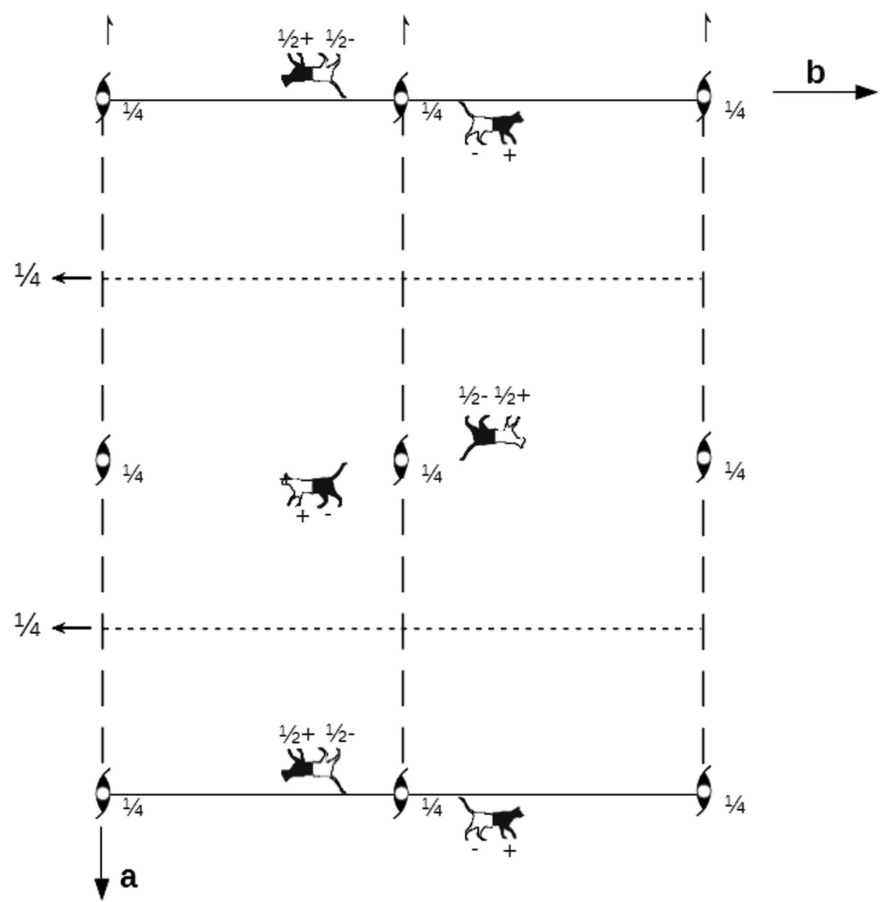

Figure 6

Result for a $\mathbf{b} / 2$ translation applied to the pattern in Fig. 5, giving an alternative but equivalent description of the same pattern.

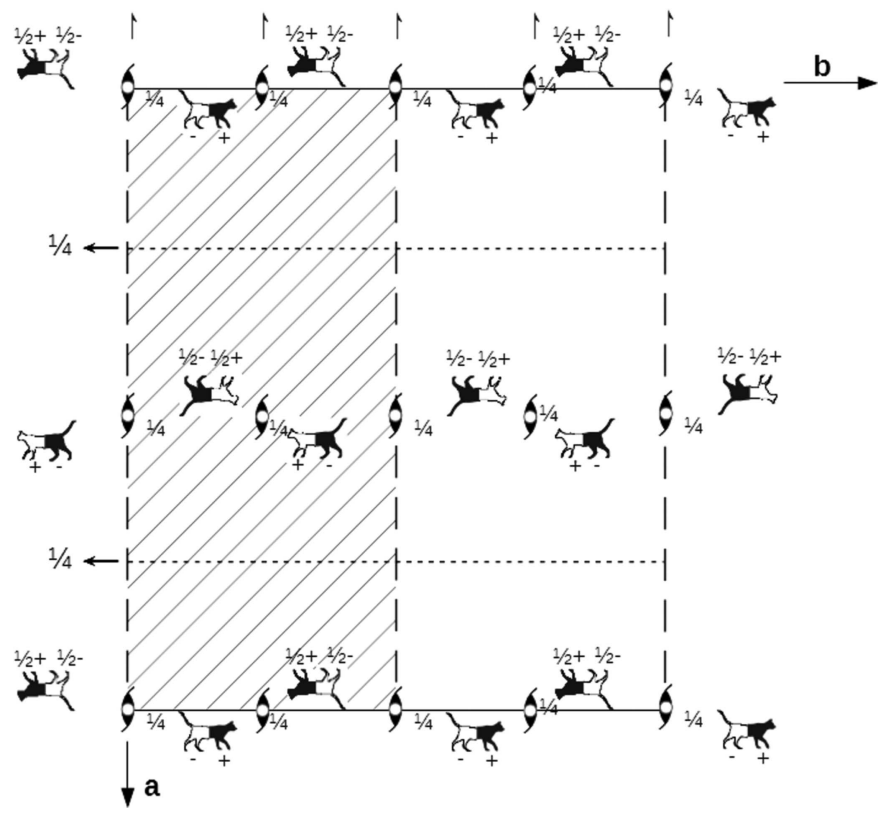

Figure 7

Overlap of the patterns in Figs. 5 and 6: the space group of this pattern is an isomorphic supergroup of $P 2_{1} / c 2 / a 2_{1} / m$ with double translational symmetry along $\mathbf{b}$. The hatching indicates the unit cell of the compound pattern. 
axes become rotation axes and the $c_{[100]}$ glide planes become mirror planes. Because of the continuous translation along $\mathbf{c}$, the Hermann-Mauguin symbol of this group is written as $P^{1} \mathrm{mmm}$, where the superscript ' 1 ' indicates the presence of

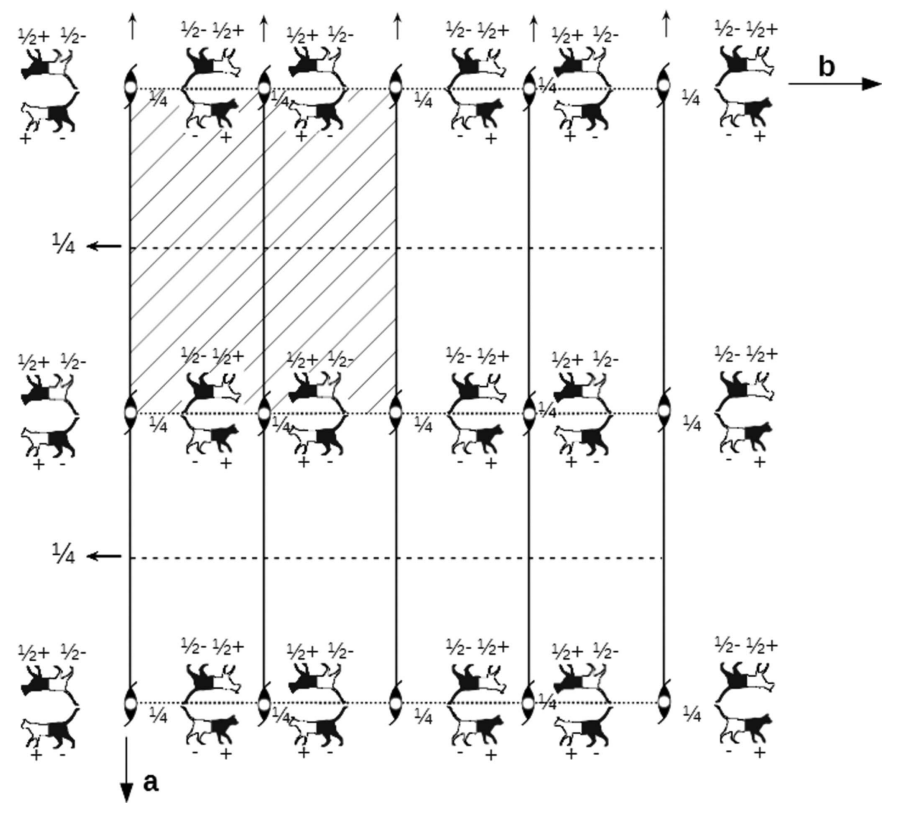

Figure 8

Overlap of the pattern in Fig. 7 with the same pattern translated by $\mathbf{a} / 2$ : the space group is $P 2 / c 2 / m 2_{1} / m(\mathbf{a} / 2, \mathbf{b} / 2, \mathbf{c})$, nonstandard setting of $P 2_{1} / m 2 / m 2 / a$, with axial transformation $\mathbf{c}, \mathbf{b},-\mathbf{a}$. The hatching indicates the unit cell of the compound pattern.

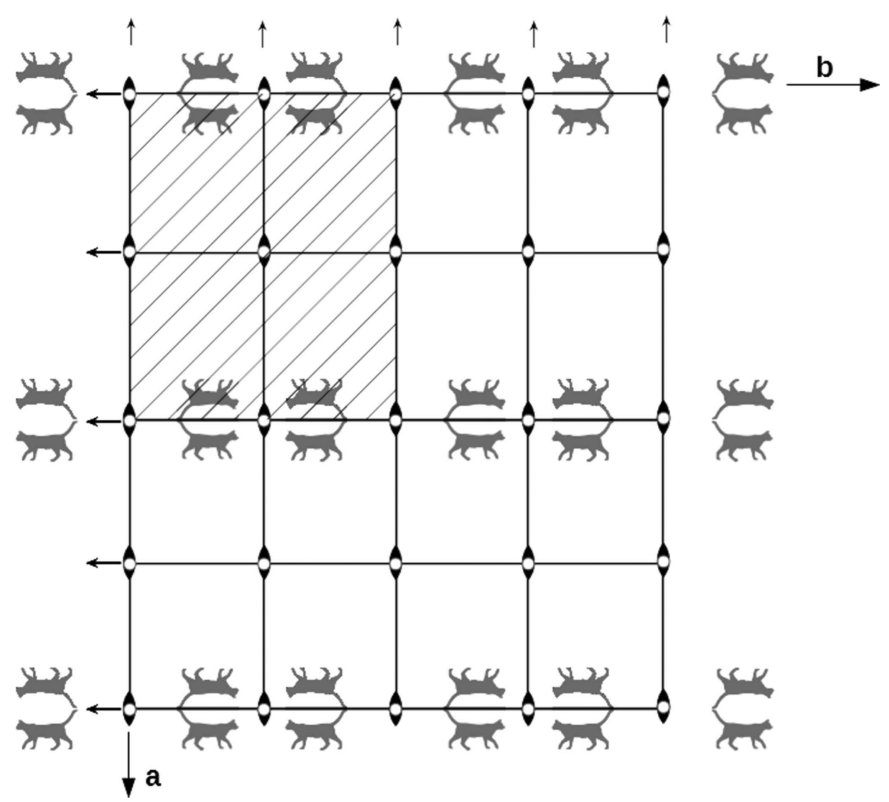

Figure 9

Overlap of an infinite number of patterns obtained by infinitesimal translations along $\mathbf{c}$ of the pattern in Fig. 8. The hatching indicates the unit cell of the compound pattern. The infinitesimal translation comes from the pyroelectric nature of $P c a 2_{1}$, which possesses a polar direction (the $c$ axis) along which the origin is not fixed a priori. The grey colour stands for black and white at the same time. The Euclidean normalizer that corresponds to this pattern is $P^{1} \mathrm{mmm}$, where the superscript ' 1 ' indicates the presence of infinitesimal translations along one direction. infinitesimal translations along one direction. This is therefore not a space group. The basis vectors of the normalizer with respect to those of the space group are $\mathbf{a} / 2, \mathbf{b} / 2, \varepsilon \mathbf{c}$, where $\varepsilon$ indicates an infinitesimal quantity coming from the continuous translation along c. Accordingly, with respect to the original group $P c a 2_{1}$ we have a series of additional generators in the normalizer: translations of $\frac{1}{2} 00,0 \frac{1}{2} 0,00 t$ ( $t$ being any value) which correspond to the shorter basis vectors, as well as an inversion at the origin (and at any value of $z$ because of the continuous translation along $\mathbf{c}$ ).

The consequence is that a crystal pattern in $P c a 2_{1}$ can receive an infinite number of equivalent descriptions. 'Equivalent' means that they differ according to the position of the observer. If we take any two of them and look at each separately, we do not see any physically relevant difference: it is only by comparing two descriptions that we recognize the difference. This corresponds to taking two feline orbits compatible with $\mathrm{Pca}_{1}$ out of the feline continuity in Fig. 9 and looking at them separately, as for the patterns in Figs. 3 and 4, or those obtained by an $\mathbf{a} / 2$ or $\mathbf{b} / 2$ translation, or by any translation along c. It is only by comparing the two patterns with a fixed position of the observer that we realize they are physically distinct: by looking at each one of them alone, they look exactly the same once a suitable position for the observer has been chosen.

\subsection{Equivalent descriptions of crystal structures}

After solving and refining the structure of a crystal from diffraction data, the structural crystallographer has to check whether the same crystal structure has already been published in the literature. In the negative case, he/she may be dealing with a new compound, or a new polymorph of a known compound. The problem is that in general the same crystal structure may be described in many different ways; in other words, the mismatch of cell parameters and/or of fractional coordinates does not necessarily mean that the structure under investigation is unknown.

One obvious reason for the existence of alternative descriptions of a crystal structure is that a space group can be described in various settings differing in the choice of the axes, the origin and the type of unit cell. ITA4 and ITA5 give some of the transformations from the standard to various nonstandard settings of space groups, although the information there is not exhaustive. The fact that the same structure gets a different description in different settings of the same space group is perfectly intuitive and does not normally represent an obstacle, even for the beginner structural investigator.

On the other hand, the existence of various alternative but equivalent descriptions of the same crystal structure in the same space group seems counter-intuitive and is surprisingly far from being a well known fact even among experienced structural investigators. Indeed, while it is obvious that any operation of the space group of a structure leaves that structure invariant, the existence of other operations that produce an undistinguishable copy of the same structure is less evident. The mechanism has been intuitively illustrated by the construction of the feline pattern in the previous section. We 
can now put the picture in a slightly more formal presentation and apply it to some examples of crystal structures.

The operations of the Euclidean group $\mathrm{E}(n)-i$.e. the whole set of isometries of the empty space - applied to a given crystal structure produce an infinite number of copies of that structure, in all possible orientations and positions, without any deformation. A subset of those operations leave the structure invariant: they form the space group of the structure, $\mathcal{G}$. An intermediate set $\mathrm{N}_{\mathrm{E}}(\mathcal{G})$, between $\mathrm{E}(n)$ and $\mathcal{G}$, produce a finite (or infinite, when $\mathcal{G}$ is a pyroelectric group) number of copies of that structure (including the original one), which do differ in the orientation and/or position in space. When looking at each of these copies taken in isolation from the others, two cases arise, depending on the chirality of the structure and the nature of its space group (Nespolo, 2015b):

(1) The difference between any pair of these copies does not have any physical or chemical meaning, like the various feline patterns in $P c a 2_{1}$ discussed above. This happens

(1.1) when the structure is achiral (its space group contains operations of the second kind);

(1.2) when both the structure and its space group are chiral: the normalizer then contains only operations of the same kind (if it is a space group, then it belongs to one of the 65 Sohncke types).

(2) Half of the copies are exactly equivalent, as in the previous case, whereas the other half correspond to the opposite absolute structure; this happens when the structure is chiral but its space group is not, i.e. the space group belongs to one of the 43 achiral Sohncke types and its normalizer contains also operations of the second kind.

Cases 1.1 and 1.2 share the common feature of possessing the same kind of operations (both first and second kind in 1.1; only first kind in 1.2) in both the space group and its normalizer. Accordingly, the operations in $\mathrm{N}_{\mathrm{E}}(\mathcal{G})$ do not modify the handedness of the crystal structure, either because it does not possess a handedness (being achiral) or because all the operations in $\mathrm{N}_{\mathrm{E}}(\mathcal{G})$ keep the handedness. In this case, the difference between the original structure $S$ and the structure $S^{\prime}$ obtained by the action of $\mathrm{N}_{\mathrm{E}}(\mathcal{G})$ simply corresponds to moving the observer around $\mathrm{S}$ until it looks exactly like $\mathrm{S}^{\prime}$.

In contrast, in case $2 \mathcal{G}$ contains only handedness-preserving operations but $\mathrm{N}_{\mathrm{E}}(\mathcal{G})$ contains also handedness-reversing operations, so that half of the copies have the same absolute structure as the original one (the same conclusion above applies again) and the other half the opposite absolute structure.

Summarizing, acting with the operations of $\mathrm{N}_{\mathrm{E}}(\mathcal{G})$ which do not belong to $\mathcal{G}$ we get a different set of fractional coordinates for the same structure. The result is either the opposite absolute structure (half of the time, in the case of chiral structures with Euclidean normalizers containing operations of the second kind) or equivalent to keeping the structure fixed and moving the observer around it. In the following, we illustrate these conclusions with a few examples chosen from structures with small unit cells, to make their visual representation easily understandable. The codes identifying the structures correspond to the Cambridge Structural Database
(CSD: Allen, 2002) and the Inorganic Crystal Structure Database (ICSD: Allmann \& Hinek, 2007).

3.2.1. Achiral structures. S-Methyl 5-methylpyrazine-2carbothioate (Aubert et al., 2007; CSD code SIMHIG) crystallizes in $\mathcal{G}=P 2_{1} / n$ (second cell choice of $P 2_{1} / c$ ) and is shown in Fig. 10(a). The Euclidean normalizer of $P 2_{1} / n$ is $\mathrm{N}_{\mathrm{E}}(\mathcal{G})=$ $P 2_{1} / m$ with basis vectors $\mathbf{a} / 2, \mathbf{b} / 2, \mathbf{c} / 2$ with respect to those of $\mathcal{G}$. The half translation along each of the axes, and thus also along the face and body diagonals, makes the glide component of the $n$ glide plane in $\mathcal{G}$ a full translation in $\mathrm{N}_{\mathrm{E}}(\mathcal{G})$, so that it changes from $n$ in $\mathcal{G}$ to $m$ in $\mathrm{N}_{\mathrm{E}}(\mathcal{G})$. The index of $\mathcal{G}$ in $\mathrm{N}_{\mathrm{E}}(\mathcal{G})$ is 8 , which means that there exist eight equivalent descriptions of the

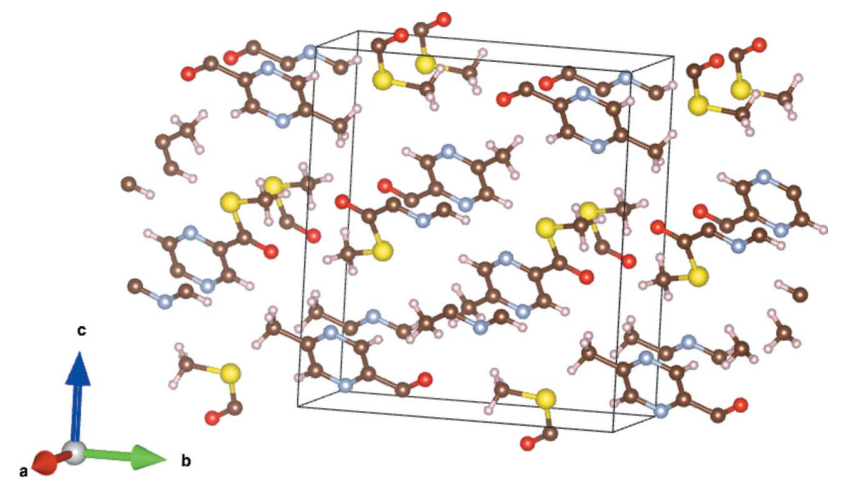

(a)

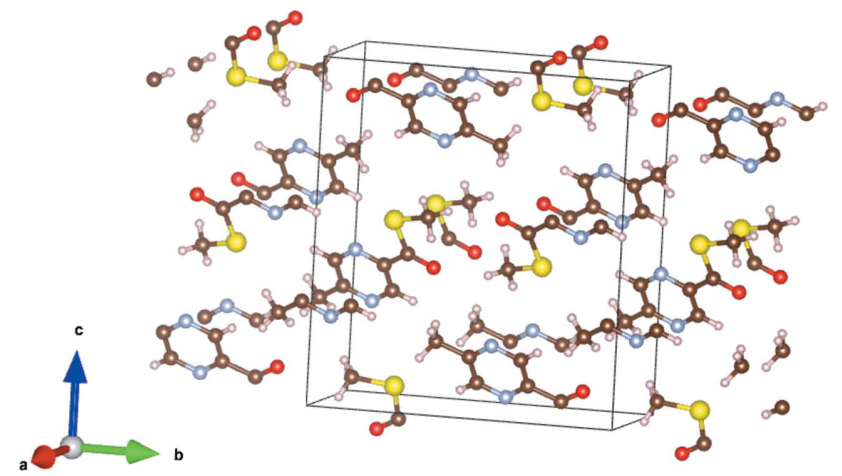

(b)

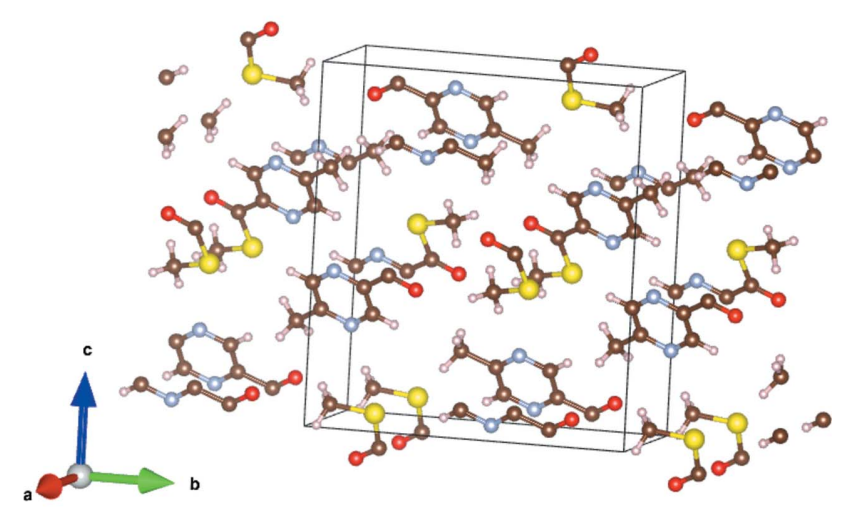

(c)

Figure 10

Structure of $S$-methyl 5-methylpyrazine-2-carbothioate. (a) Original structure. (b) The same structure translated by b/2. (c) Once again, the same structure translated by $(\mathbf{a}+\mathbf{b}) / 2$. These translations belong not to the space-group symmetry of the crystal but to its Euclidean normalizer and produce alternative but equivalent descriptions of the structure. Figure drawn with VESTA (Momma \& Izumi, 2011). 
same structure. The additional generators of $\mathrm{N}_{\mathrm{E}}(\mathcal{G})$ with respect to $\mathcal{G}$ are simply the translations $\frac{1}{2}, 0,0 ; 0, \frac{1}{2}, 0$; and $0,0, \frac{1}{2}$. Figs. $10(b)$ and $10(c)$ show the structure translated by $\mathbf{b} / 2$ and $(\mathbf{a}+\mathbf{b}) / 2$, respectively. While at first sight the three structures may seem different, they actually differ in the position of the observer, moved by translation.

Caesium acetate, $\mathrm{Cs}\left(\mathrm{CH}_{3} \mathrm{COO}\right)$, crystallizes in a space group of type P6/m (Lossin \& Meyer, 1993; CSD code LASSUT); the structure is shown in Fig. 11( $a)$. The Euclidean normalizer of $P 6 / \mathrm{m}$ is $\mathrm{N}_{\mathrm{E}}(\mathcal{G})=P 6 / \mathrm{mmm}$ with basis vectors $\mathbf{a}, \mathbf{b}$, c/ 2 with respect to those of $\mathcal{G}$. The index of $\mathcal{G}$ in $\mathrm{N}_{\mathrm{E}}(\mathcal{G})$ is 4 and the latter contains two additional generators, of which one is a half-period translation along $\mathbf{c}$ and produces a translated structure, as in the previous case. The second generator is the mirror plane $m x, x, z$. Fig. 11(b) shows the structure of caesium acetate transformed by the action of this generator. At first glance, the two structures may appear different. To show that this is not the case, one simply needs to change the position of the observer. $\mathcal{G}$ is centrosymmetric and the structure is achiral, so that there must be an isometry of the first kind which produces the same result as the reflection across $m$ $x, x, z$. Indeed, by decomposing $\mathrm{N}_{\mathrm{E}}(\mathcal{G})$ with respect to $\mathcal{G}$ one gets three cosets, each of which contains as many operations of the first kind as those of the second kind. In the same coset containing $m x, x, z$ we find a twofold rotation about [110]. If

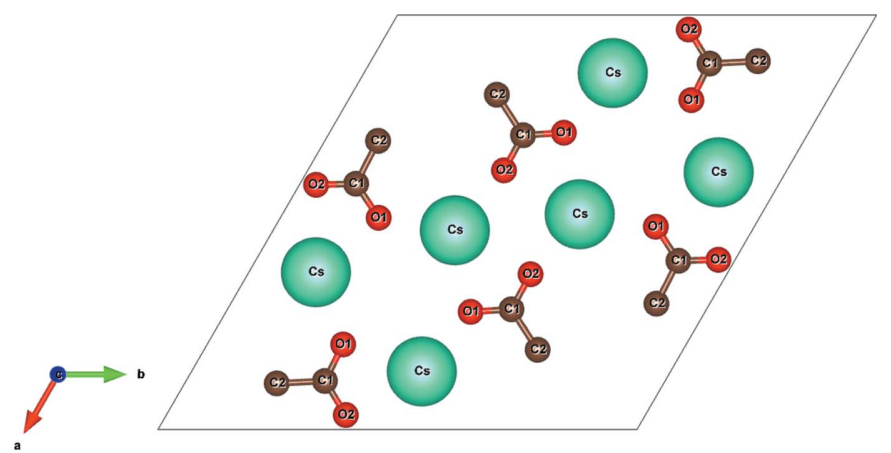

(a)

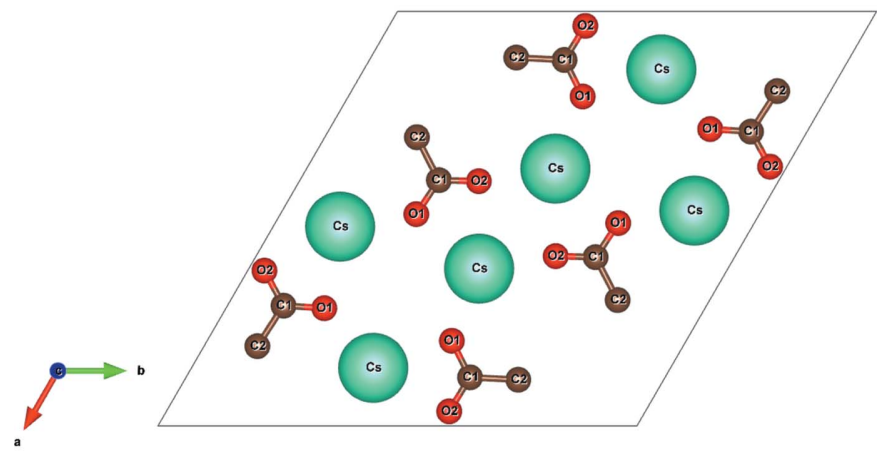

(b)

Figure 11

Structure of caesium acetate. (a) Original structure. (b) The same structure transformed by the $m x, x, z$ operation, which is an additional generator of the Euclidean normalizer of the space group of the structure. The equivalence of figures $(a)$ and $(b)$ can be seen by looking at $(a)$ from a different position, corresponding to a $2 x, x, 0$ rotation applied to the axial setting, or by looking at $(b)$ from the reverse of the page and rotating it $120^{\circ}$ counterclockwise. Figure drawn with VESTA. now we apply this rotation to Fig. 11(a) - not to the fractional coordinates of the atoms but to the figure itself - we get Fig. 11(b): this corresponds to moving the observer to the opposite side from where he/she was looking at Fig. 11(a). Fig. 11(a) (the original structure) and Fig. 11(b) (the structure on which the normalizer has acted) differ in the labelling of the axes, the transformation being ( $\mathbf{a b c})$ to $(\mathbf{b a \mathbf { c }})$, i.e. precisely the $2 x, x, 0$ rotation applied to the axial setting. This operation does belong to the symmetry of the lattice (the holohedry) and the difference between the two figures is really just in the labels of the axes.

3.2.2. Chiral structures with a chiral space group. This case is the easiest to understand, as it can be shown by the example of $\gamma$-glycine (Shimon et al., 1986; CSD code GLYCIN18), which crystallizes in a space group of type $P 3_{1}$, whose Euclidean normalizer is $P^{1} 622$, with basis vectors $\frac{2}{3} \mathbf{a}+\frac{1}{3} \mathbf{b},-\frac{1}{3} \mathbf{a}+\frac{1}{3} \mathbf{b}$, $\varepsilon$ c with respect to those of $\mathcal{G}$; the additional generators in $\mathrm{N}_{\mathrm{E}}(\mathcal{G})$ with respect to $\mathcal{G}$ are the translation $\frac{21}{33} 0$, the infinitesimal translation $00 t$, and a twofold rotation about [100] and a twofold rotation about $\langle 110\rangle$, both passing through the origin (ITA15).

Fig. 12(a) shows the original structure (S) and Fig. 12(b) a copy $\left(\mathrm{S}^{\prime}\right)$ obtained by applying the $20,0, z$ rotation to the fractional coordinates. If we observe Fig. 12(a) from the negative side of the [110] direction, i.e. by moving the observer $180^{\circ}$ around the $c$ axis, we get Fig. 12(b). Clearly, the difference between $S$ and $S^{\prime}$ is, as before, in the position of the observer.

3.2.3. Chiral structures with a normalizer containing operations of the second kind. $\beta-\mathrm{NbO}_{2}$ (Schweizer \& Gruehn, 1982; ICSD code 35181) crystallizes in a space group of type $I 4_{1}$, one of the 65 Sohncke group, whose normalizer is $P^{1} 4 / n b m$, with basis vectors $\frac{1}{2}(\mathbf{a}-\mathbf{b}), \frac{1}{2}(\mathbf{a}+\mathbf{b}), \varepsilon \mathbf{c}$ with respect to those of $\mathcal{G}$; the additional generators in $\mathrm{N}_{\mathrm{E}}(\mathcal{G})$ with respect to $\mathcal{G}$ are the infinitesimal translation $00 t$, a $2_{\langle 110\rangle}$ rotation and an inversion through the point $\frac{1}{4}, 0,0$. The first two generators do not add anything new with respect to the cases analysed above, the difference between $S$ and $S^{\prime}$ being in the position of the observer. The third generator, instead, inverts the absolute structure (Figs. $13 a$ and $13 b$ ), a result that cannot be equivalently obtained by moving the observer around. Note that the position of the inversion centre in the Euclidean normalizer is of paramount importance when a wrong absolute structure comes out of the refinement process (value of the Flack parameter close to 1; Flack, 2003). Although it is often at the origin of the normalizer, this is not always the case, as shown by the example of $\beta-\mathrm{NbO}_{2}$. By simply inverting the sign of the fractional coordinates of each atom, as if the centre of inversion were at the origin of the Euclidean normalizer, one would get a wrong disconnected structure (Fig. 13c).

\section{When (some) deformations are forgiven: affine normalizers}

Instead of the Euclidean group $\mathrm{E}(n)$, one may use a more general group to produce copies of one's structure. This larger group is the affine group $\mathrm{A}(n)$, i.e. the group of all invertible 
affine transformations from the space into itself. An affine transformation is a function which preserves points, straight lines and planes so that sets of parallel lines remain parallel after an affine transformation; while it does preserve ratios of distances between points lying on a straight line, it does not necessarily preserve angles between lines or distances between points. In other words, an affine transformation is a special case of deformation, which allows metric specialization and is of special importance in crystallography, because more often than one may expect crystal structures have a metric symmetry corresponding to a higher holohedry than their structural symmetry (Janner, 2004a,b). If that is the case, then the use of the Euclidean normalizer is not enough to produce all the equivalent descriptions of the given crystal structure and the affine normalizer has to be considered instead. The affine normalizer does not depend on the metrical properties of the space group, as is instead the case for the Euclidean normalizer; depending on the metric specializations a space group may have more than one Euclidean normalizer and the normalizer corresponding to the highest symmetry is the affine normalizer. Clearly, if the metric specialization does not lead to any increase in the symmetry of the normalizer, than the affine and the (only) Euclidean normalizers coincide. For example, a tetragonal space group has only a single fourfold axis, even if $c=a$, and this axis is not fixed by a fourfold rotation about [100] or [010]. Thus, a cubic specialization of a tetragonal space group does not have consequences on the normalizer of that group, which remains tetragonal.

Space groups are classified in terms of their normalizers as follows (ITA15):

(1) Cubic, hexagonal, trigonal and tetragonal space groups, as well as 21 types of orthorhombic space group, have only one type of Euclidean normalizer, which also coincides with the affine normalizer.

(2) The other 38 types of orthorhombic space group have more than one Euclidean normalizer, as a function of the metric specialization; the affine normalizer coincides with the highest-symmetry Euclidean normalizer.

(3) Affine normalizers of monoclinic and triclinic space groups are not isomorphic to any group of motions and cannot be characterized by a space-group symbol.

The case of orthorhombic groups can be illustrated by the examples of Pnnm (No. 58), Pnna (No. 52) and Pbca (No. 61). The first of these groups, whose full symbol is $P 2_{1} / n 2_{1} / n 2 / m$, has the same type of symmetry elements (twofold screw axes and $n$ glide planes) along [100] and [010] and a mirror plane

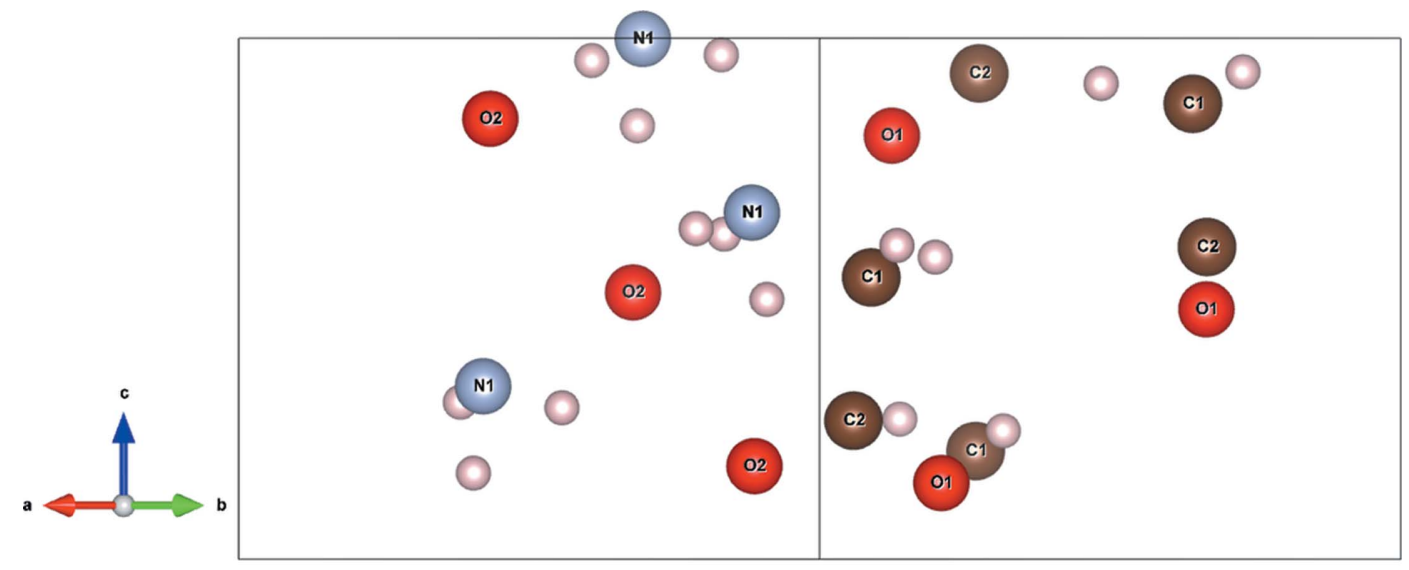

(a)

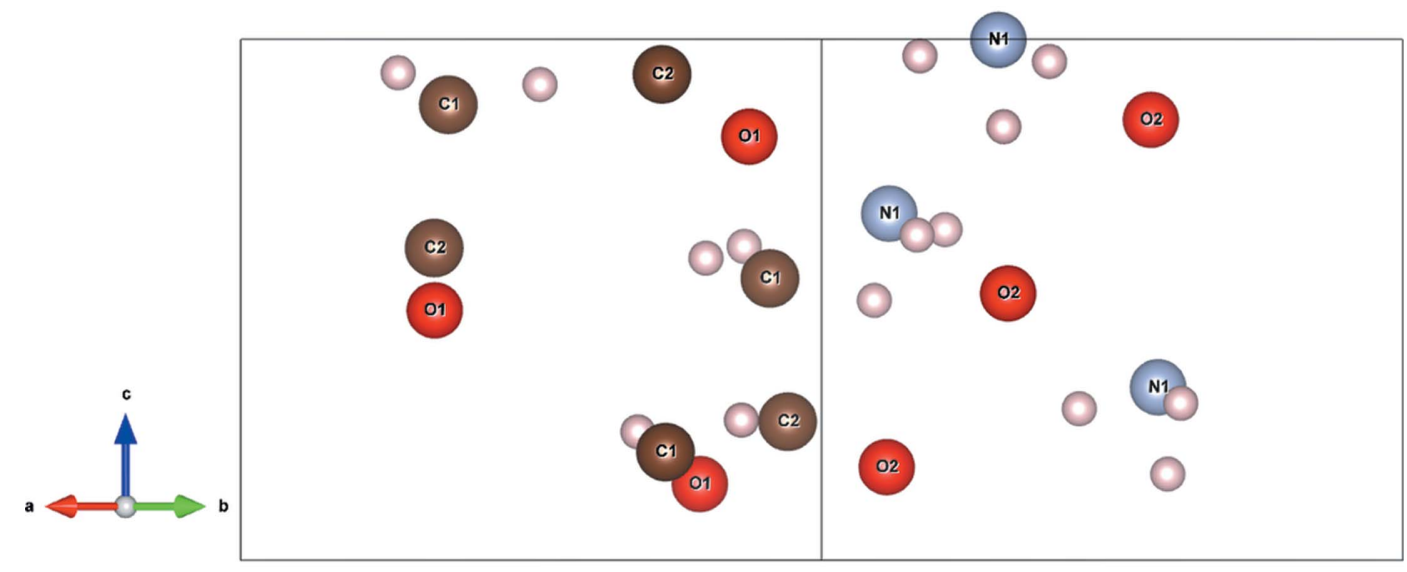

(b)

Figure 12

Structure of $\gamma$-glycine. (a) Original structure. (b) Result of applying a $20,0, z$ rotation to the fractional coordinates in $(a)$. If $(a)$ is observed from the negative side of the [110] direction, i.e. by moving the observer $180^{\circ}$ around the $c$ axis, one gets $(b)$. Figure drawn with $V E S T A$. 
normal to [001]. In the case of metric specialization $a=b$ the group is self-conjugated by a fourfold rotation about [001], and its Euclidean normalizer for this specialization is $P 4 / \mathrm{mmm}$ with basis vectors $\mathbf{a} / 2, \mathbf{b} / 2, \mathbf{c} / 2$ with respect to those of Pnnm: additional generators the half-translations and the $y x z$ reflection (mirror normal to [110]). A further metric specialization $a=b=c$ does not lead to a higher-symmetry normalizer because Pnnm does not contain the same type of symmetry element along the three axes. Therefore, the affine normalizer of Pnnm coincides with the Euclidean normalizer for the case $a=b$. A structure crystallizing in Pnnm has eight equivalent descriptions for the general metric (differing for the half-translation along each of the axes or diagonals), but 16 in the case of the tetragonal metric specialization $a=b$ (the translations above plus the operations in the coset obtained by the $m x x z$ generator).

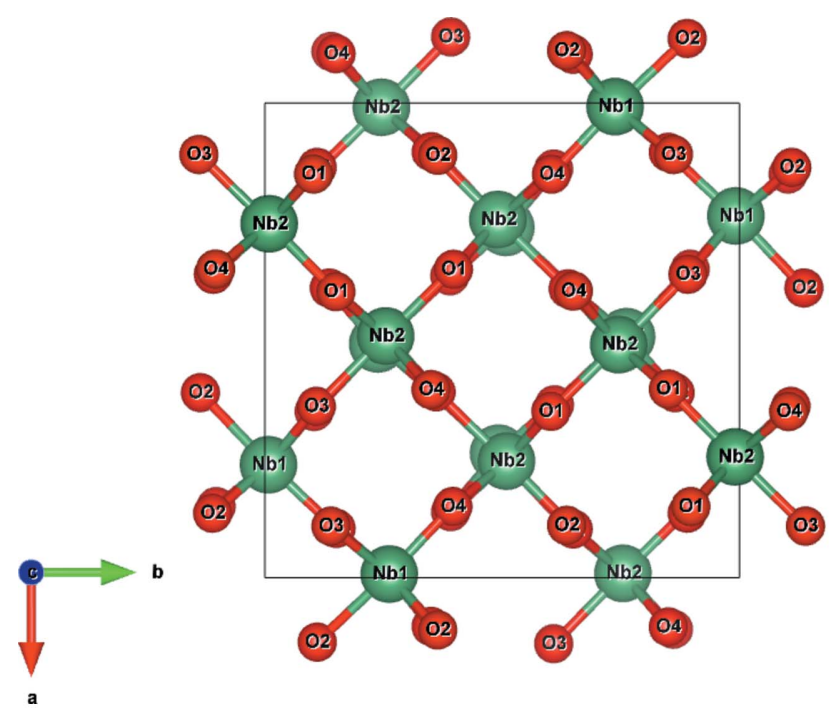

(a)
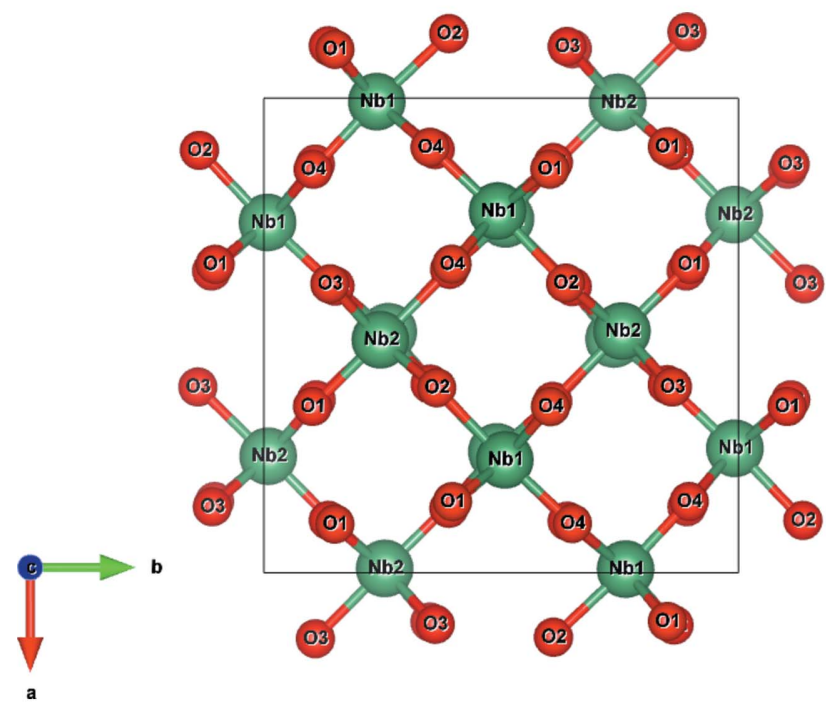

(b)
Pnna is instead one of the 21 types of orthorhombic spacegroup types having only one Euclidean normalizer. This group, whose full symbol is $P 2 / n 2_{1} / n 2 / a$, is not self-conjugated by a fourfold rotation about [001] even if $a=b$. This is because it has a twofold axis along [100] but a twofold screw axis along [010] as well as an $a$ glide perpendicular to [001], which makes the two directions [100] and [010] non-equivalent even in the case of tetragonal metric specialization. Therefore, Pnna has only one Euclidean normalizer, Pmmm (basis vectors of the normalizer: $\mathbf{a} / 2, \mathbf{b} / 2, \mathbf{c} / 2$ ), which is also its affine normalizer. A structure crystallizing in Pnna has eight equivalent descriptions (obtained by the half-translations) independently of any metric specialization.

$P b c a$, whose full symbol is $P 2_{1} / b 2_{1} / c 2_{1} / a$, has the same type of symmetry elements (twofold screw axes) along the three axes and perpendicular to them glide planes whose glide components permute along one of the in-plane axes. In the case of tetragonal metric specialization the Euclidean normalizer is still orthorhombic (Pmmm with basis vectors $\mathbf{a} / 2$, $\mathbf{b} / 2, \mathbf{c} / 2)$ because the glide plane normal to the fourfold axis makes the two in-plane directions non-equivalent, as in the previous case. However, in the case of cubic metric specialization, each of the three axes comes to play the same role, so that $P b c a$ is self-conjugated under $P m \overline{3}$, which is the Euclidean normalizer for this specialized metric (basis vectors again $\mathbf{a} / 2, \mathbf{b} / 2, \mathbf{c} / 2)$ as well as the affine normalizer for Pbca. A structure crystallizing in $\mathrm{Pbca}$ has eight equivalent descriptions for the general metric or for any tetragonal specialization (obtained by the half-translations), but 24 in the case of cubic metric specialization (the translations above plus the operations in the coset obtained by the $3 x x x$ generator).

Fig. 14(a) shows the structure of cuproan adamite, $\mathrm{Zn}\left(\mathrm{Zn}_{0.16} \mathrm{Cu}_{0.84}\right)\left(\mathrm{AsO}_{4}\right)(\mathrm{OH})$ (Toman, 1978; H-atom positions

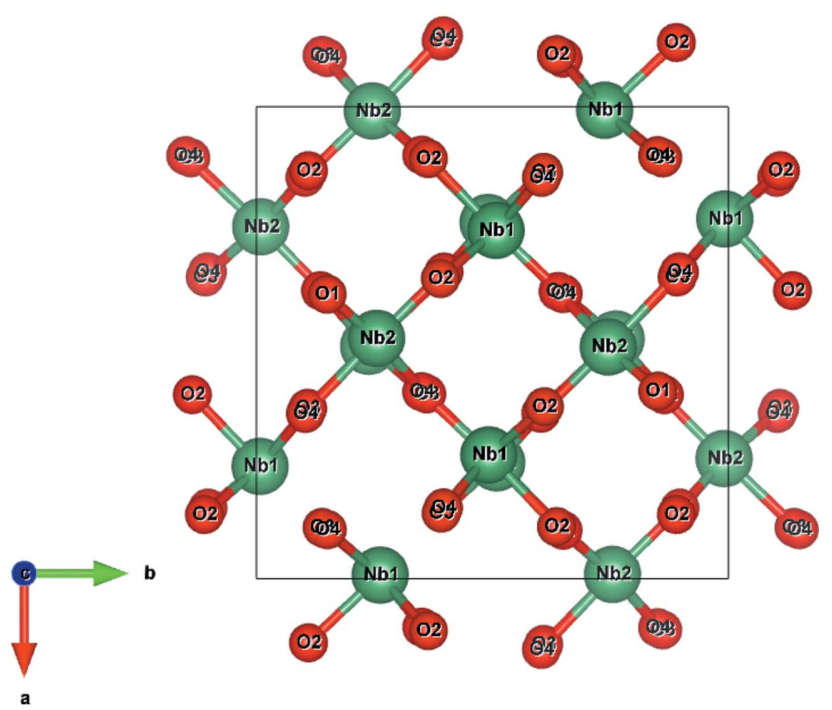

(c)

Figure 13

Structure of $\beta-\mathrm{NbO}_{2}$. (a) Original structure. (b) Result of the application of an inversion through the point $\frac{1}{4}, 0,0$, which is an additional generator in the Euclidean normalizer: the opposite absolute structure is obtained. $(c)$ A wrong disconnected structure obtained by simply inverting the signs of the fractional coordinates, as could be mistakenly done when trying to get the correct absolute structure without considering the position of the inversion centre in the normalizer. Figure drawn with VESTA. 

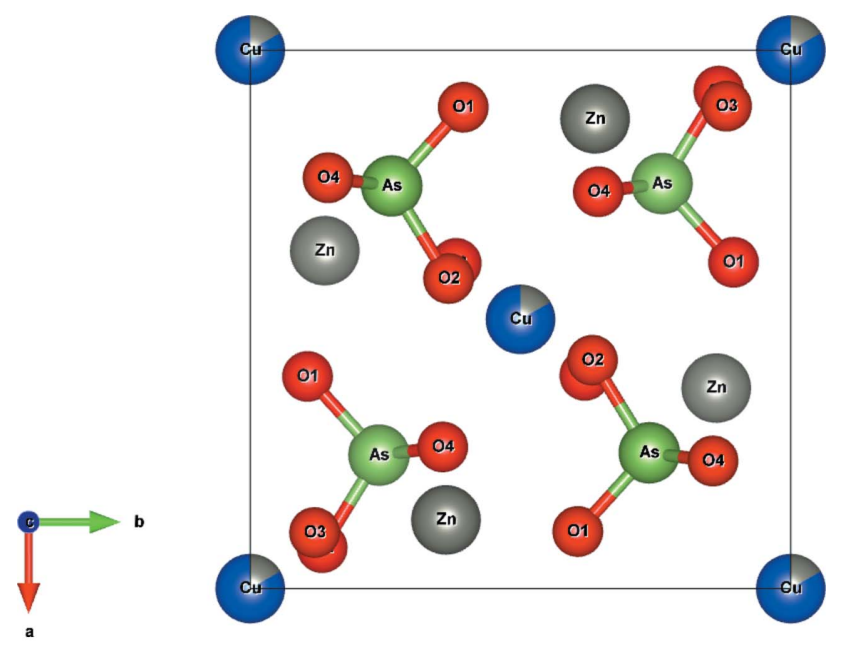

(a)
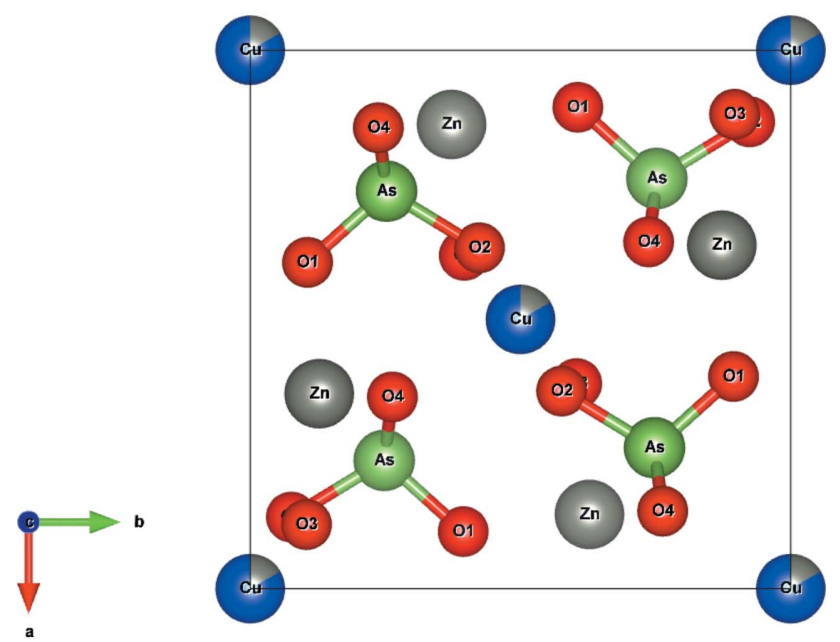

(b)

Figure 14

Structure of cuproan adamite, which possesses a tetragonal specialized metric. (a) Original structure. The position at the origin is occupied by $\mathrm{Cu}$ and $\mathrm{Zn}$ (86 and 14\%, respectively); the label of the more abundant atom is shown in the figure. $(b)$ The same structure transformed by applying an $m x x z$ reflection, which is an additional generator of the affine normalizer. If one looks from the negative [001] direction with a $90^{\circ}$ counterclockwise orientation one gets $(b)$. Figure drawn with VESTA.

undetermined; ICSD code 1516), which crystallizes in Pnnm, $a=8.50$ (2), $b=8.52$ (2), $c=5.99$ (1) А, with a specialized tetragonal metric within the standard uncertainty on the cell parameters. As discussed above, the affine normalizer is tetragonal with additional generator $m x x z$. Fig. $14(b)$ is the same structure transformed by applying this operation, i.e. $x y z \rightarrow y x z$. Fig. 14(b) is obtained also from Fig. 14(a) through a $2_{\text {[110] }}$ rotation, which corresponds to moving the observer behind the plane of the original figure and turning him or her $90^{\circ}$ counterclockwise. The two descriptions of the structure are indistinguishable.

\section{Discussion}

The description of crystal structures by their symmetry groups is a useful abstraction, by which the crystallographer gives an idealized (time- and space-averaged) model in which static and dynamic defects are ignored. In order to make 'the same symmetry group' actually look the same, standard settings are agreed on for the 230 types of crystallographic space groups which give a unique configuration of symmetry elements, abstracting them from alternative settings differing, for example, in the axes labels and the origin choices (which increase significantly that number). However, this configuration is not rigid but still allows transformations by some motions, which are the elements of the normalizer; the result is a set of equivalent descriptions of a crystal structure. This is the most evident use of normalizers, which however intervene in diverse tasks such as the solution of the ambiguities in direct methods (ITA15) and the derivation of the effect of merohedric twinning on the reflection conditions (Nespolo et al., 2014). Often wrongly considered an 'esoteric' topic left outside the standard education of a crystallographer, it is actually of fundamental help in structural investigation. We hope that this short survey will be useful to make normalizers more widely known and appreciated.

\section{Acknowledgements}

This research has been partly conducted during a stay as invited professor at Kyoto University. Helpful remarks by two anonymous reviewers are thankfully acknowledged and appreciated.

\section{References}

Allen, F. H. (2002). Acta Cryst. B58, 380-388

Allmann, R. \& Hinek, R. (2007). Acta Cryst. A63, 412-417.

Aroyo, M. I., Perez-Mato, J. M., Capillas, C., Kroumova, E., Ivantchev, S., Madariaga, G., Kirov, A. \& Wondratschek, H. (2006). Z. Kristallogr. 221, 15-27.

Aubert, E., Mamane, V. \& Fort, Y. (2007). Acta Cryst. E63, o4306-o4307.

Flack, H. (2003). Helv. Chim. Acta, 86, 905-921.

Hahn, Th. (2005). Editor. International Tables for Crystallography, Vol. A, Space-Group Symmetry, 5th ed. Heidelberg: Springer.

Janner, A. (2004a). Acta Cryst. A60, 198-200.

Janner, A. (2004b). Acta Cryst. A60, 611-620.

Koch, E. \& Fischer, W. (2006). Z. Kristallogr. 221, 1-14.

Lossin, A. \& Meyer, G. (1993). Z. Anorg. Allg. Chem. 619, 1462-1464.

Momma, K. \& Izumi, F. (2011). J. Appl. Cryst. 44, 1272-1276.

Nespolo, M. (2015a). J. Appl. Cryst. 48, 1290-1298.

Nespolo, M. (2015b). Cryst. Res. Technol. 50, 413.

Nespolo, M. \& Aroyo, M. I. (2015). Eur. J. Mineral. doi:10.1127/ejm/ 2015/0027-2492.

Nespolo, M., Ferraris, G. \& Souvignier, B. (2014). Acta Cryst. A70, 106-125.

Nespolo, M. \& McColm, G. (2014). Acta Cryst. A70, 317-318.

Sadanaga, R., Sawada, T., Ohsumi, K. \& Kamiya, K. (1980). J. Jpn. Assoc. Min. Petr. Econ. Geol. Spec. Issue No. 2, 23-29.

Schweizer, H. J. \& Gruehn, R. (1982). Z. Naturforsch. Teil B, 37, 1361-1368.

Shimon, L. J. W., Lahav, M. \& Leiserowitz, L. (1986). New J. Chem. 10, 723-737.

Tasci, E. S., de la Flor, G., Orobengoa, D., Capillas, C., Perez-Mato, J. M. \& Aroyo, M. I. (2012). EPJ Web Conf. 22, 00009.

Terpstra, P. \& Codd, L. W. (1961). Crystallometry. New York: Academic Press.

Toman, K. (1978). Acta Cryst. B34, 715-721.

Whittaker, E. J. W. (1984). The Stereographic Projection. IUCr Teaching Pamphlet No. 11, http://www.iucr.org/education/ pamphlets/11. 